\title{
Quantum Error Correction with the Gottesman-Kitaev-Preskill Code
}

\author{
Arne L. Grimsmo $\oplus^{1}$ and Shruti Puri ${ }^{2, *}$ \\ ${ }^{1}$ ARC Centre of Excellence for Engineered Quantum Systems, School of Physics, The University of Sydney, \\ Sydney, NSW 2006, Australia \\ ${ }^{2}$ Department of Applied Physics, Yale University, New Haven, Connecticut 06511, USA
}

(Received 27 April 2021; published 23 June 2021)

\begin{abstract}
The Gottesman-Kitaev-Preskill (GKP) code was proposed in 2001 by Daniel Gottesman, Alexei Kitaev, and John Preskill as a way to encode a qubit in an oscillator. The GKP codewords are coherent superpositions of periodically displaced squeezed vacuum states. Because of the challenge of merely preparing the codewords, the GKP code was for a long time considered to be impractical. However, the remarkable developments in quantum hardware and control technology in the last two decades has made the GKP code a frontrunner in the race to build practical, fault-tolerant bosonic quantum technology. In this Perspective, we provide an overview of the GKP code with emphasis on its implementation in the circuit-QED architecture and present our outlook on the challenges and opportunities for scaling it up for hardware-efficient, fault-tolerant quantum error correction.
\end{abstract}

DOI: 10.1103/PRXQuantum.2.020101

\section{INTRODUCTION}

In 2001, Gottesman, Kitaev, and Preskill published a proposal to encode discrete quantum information in a continuous-variable quantum system, or in other words, "a qubit in an oscillator" [1]. The encoding is designed such that it is possible to correct small shifts in the position and momentum quadratures of the oscillator. This remarkable idea can safely be said to have been ahead of its time: It took almost 20 years before experimental realization of their proposal was made by the Home group using an oscillating trapped ion [2]. It was almost immediately followed by the experimental realization in the Devoret group, using a microwave cavity and a circuit quantum electrodynamics (cQED) approach [3]. More broadly, these two experiments are part of a flourishing effort to demonstrate robust encoding of quantum information using bosonic degrees of freedom, with the ultimate long-term goal of building a fault-tolerant quantum computer [4-10].

The idea of encoding information in a continuousvariable quantum system is in many ways very natural. Quantum harmonic oscillators abound in nature, and well-defined bosonic modes can be isolated from environmental noise in many quantum technology platforms.

\footnotetext{
${ }^{*}$ r.shruti.puri@gmail.com

Published by the American Physical Society under the terms of the Creative Commons Attribution 4.0 International license. Further distribution of this work must maintain attribution to the author(s) and the published article's title, journal citation, and DOI.
}

Early proposals for bosonic error-correcting codes were made already in the late 1990s [11,12]. The core idea behind these proposals is to encode $k$ logical qubits into $n$ bosonic modes, and attempt to exploit the large Hilbert space of each bosonic mode to achieve an efficient encoding with good error-correcting properties for a small $n$. Successful bosonic codes are referred to as hardware efficient. Remarkably, interesting bosonic codes, and the Gottesman-Kitaev-Preskill (GKP) code in particular, exist even for $k=n=1$.

The primary requirement for implementing bosonic codes, and also why it took two decades of technological developments before the GKP states were realized, is control of a high-quality harmonic oscillator mode with a sufficiently strong and high-quality ancillary nonlinearity. This nonlinearity can be a discrete two-level system. In the case of trapped ions, a bosonic state is encoded in the harmonic motion of a single trapped ion by exploiting the strong coupling with the ancillary atomic pseudospin states. In the case of cQED, the ancillary levels of a transmon have been used to realize a bosonic encoding in the microwave fields of a superconducting cavity or resonator. Harmonic modes with high quality factors, combined with easy access to strong nonlinearities with minimal dissipation, lead to an unprecedented coherent control over the oscillator Hilbert space in these platforms $[2-7,10,13]$.

Optical systems are also researched actively in the context of GKP codes [14-17]. Some proposals are based on using optical nonlinearities or interaction between atoms and light to generate photonic GKP states $[18,19]$. Several other proposals rely on photon-number-resolving detectors 
as the ancillary nonlinear resource [20,21]. However, because of photon loss, weak optical nonlinearities, and the need for complex multiplexing with high-efficiency number-resolving detectors, generation of the highly nonGaussian GKP states have not yet been demonstrated in the optical domain.

Given that preparation of GKP-encoded states has now been demonstrated in the lab - and it is not a big leap to imagine that gates between two encoded GKP qubits are right around the corner - it is natural to ask whether GKP encodings can become a competitive approach to largescale, fault-tolerant quantum computing. While the GKP code can correct for small quadrature shifts in the oscillator, realistic noise in an experimental platform is more complex and can introduce uncorrectable errors. Therefore, in practice the suppression in the logical error rate with a single-mode GKP code will be limited. A natural approach to "scale up" is to reduce errors as much as possible in the single-mode encoding and then concatenate a number of encoded GKP qubits to a second error-correcting code, for example a surface code, for a total of one logical qubit across $n$ physical modes [22-25]. If this approach leads to a substantially lower logical error rate than using a comparable number of unencoded physical qubits, one may achieve a better encoding with a similar hardware cost.

A milestone towards this goal of resource-efficient fault tolerance would be to demonstrate basic operations on the encoded GKP states, used to compose error-correction circuits, with fidelities that are comparable or better than the best physical qubits to date. These operations include state preparation, entangling gates between two GKP-encoded modes, and measurement. This is a challenging goal considering the high-fidelity qubit operations in both trapped ions and superconducting qubits today.

A fundamental obstacle to achieving high-fidelity operations on encoded GKP states is that practical constructions of the required interactions can ruin the protection offered by the bosonic encoding. For example, if a two-level system is used to control the oscillator mode, a single error on the two-level system may propagate to a logical error on the mode $[2,3,10]$. This can prohibit the fidelity of encoded operations on the GKP states from being significantly better than those of the unencoded two-level ancilla. How to best achieve fault tolerance against such ancilla errors in a bosonic code architecture is an important open question, and we touch on some of the possibilities that have been put forth towards this goal.

In this Perspective, we discuss the prospect of scalable, fault-tolerant quantum computing with GKP codes with special emphasis on its implementation in a cQED architecture. While there are several excellent review articles on GKP and other bosonic codes [24,26-28], here we provide an application-level perspective highlighting the outstanding practical challenges. We focus on CQED partly because the two authors are working in this field, but also because we believe the flexibility and scalability of superconducting circuits make this a particularly promising platform for the long-term goal of constructing a large-scale quantum computer based on bosonic encodings.

With this in mind, we begin with an overview of the GKP code in Sec. II, go on to discuss state preparation and error correction in Sec. III, and address the question of fault-tolerant, scalable quantum computing with GKP codes in Sec. IV. Throughout this Perspective, we emphasize not only the advances made towards GKP error correction but also the challenges that must be overcome to make practical fault tolerance with GKP codes possible. These challenges and opportunities for future research are summarized in Sec. V.

\section{INTRODUCTION TO GOTTESMAN-KITAEV-PRESKILL CODES}

\section{A. Basic definitions}

In general, GKP codes encode a $d$-dimensional logical subspace in $n$ bosonic modes [1]. We here focus exclusively on the simplest nontrivial case $d=2$ and $n=1$, i.e., a single logical qubit encoded in a single bosonic mode. To define a GKP code, it is first convenient to introduce the displacement operators $\hat{D}(\alpha)=e^{\alpha \hat{a}^{\dagger}-\alpha^{*} \hat{a}}$, where $\left[\hat{a}, \hat{a}^{\dagger}\right]=1$ are the usual ladder operators of a harmonic oscillator and $\alpha$ is a complex number. The displacement operators satisfy the property

$$
\begin{aligned}
\hat{D}(\beta) \hat{D}(\alpha) & =e^{\left(\beta \alpha^{*}-\beta^{*} \alpha\right) / 2} \hat{D}(\alpha+\beta) \\
& =e^{\beta \alpha^{*}-\beta^{*} \alpha} \hat{D}(\alpha) \hat{D}(\beta) .
\end{aligned}
$$

In other words, displacements commute "up to a phase." In particular, if

$$
\beta \alpha^{*}-\beta^{*} \alpha=i \pi,
$$

the two operators anticommute, while if $\alpha \beta^{*}-\alpha \beta^{*}=2 i \pi$ they commute.

To define a GKP code, we first choose logical Pauli operators $\bar{X}=\hat{D}(\alpha)$ and $\bar{Z}=\hat{D}(\beta)$, where $\alpha$ and $\beta$ are any two complex numbers that satisfy Eq. (2). This ensures that $\bar{X} \bar{Z}=-\bar{Z} \bar{X}$. To ensure that $\bar{X}, \bar{Z}$, and $\bar{Y}=i \bar{X} \bar{Z}=$ $\hat{D}(\alpha+\beta)$ behave like the usual two-by-two Pauli matrices, they should also square to the identity on any state in the code subspace (codespace). We therefore define the GKP logical codespace to be the simultaneous +1 eigenspace of the two operators

$$
\hat{S}_{X}=\bar{X}^{2}=\hat{D}(2 \alpha), \quad \hat{S}_{Z}=\bar{Z}^{2}=\hat{D}(2 \beta) .
$$

It follows from Eq. (1) that these two operators commute with each other, and the logical Paulis. The set $\left\{\hat{S}_{X}^{k}, \hat{S}_{Z}^{l}\right\}$ for $k, l \in \mathbb{Z}$ form the stabilizer group of the GKP code. 
We can write the GKP codewords explicitly in terms of sums of quadrature eigenstates. To this end, first define two generalized quadratures $\hat{Q}=i\left(\beta^{*} \hat{a}-\beta \hat{a}^{\dagger}\right) / \sqrt{\pi}, \hat{P}=$ $-i\left(\alpha^{*} \hat{a}-\alpha \hat{a}^{\dagger}\right) / \sqrt{\pi}$, such that $[\hat{Q}, \hat{P}]=i$ and

$$
\bar{X}=e^{-i \sqrt{\pi} \hat{P}}, \quad \bar{Z}=e^{i \sqrt{\pi} \hat{Q}}, \quad \bar{Y}=e^{i \sqrt{\pi}(\hat{Q}-\hat{P})} .
$$

It is straight forward to check that

$$
\begin{aligned}
& \left|0_{L}\right\rangle=\sum_{j=-\infty}^{\infty}|2 j \sqrt{\pi}\rangle_{\hat{Q}}, \\
& \left|1_{L}\right\rangle=\sum_{j=-\infty}^{\infty}|(2 j+1) \sqrt{\pi}\rangle_{\hat{Q}},
\end{aligned}
$$

are \pm 1 eigenstates of $\bar{Z}$, respectively, and +1 eigenstates of $\hat{S}_{X}$ and $\hat{S}_{Z}$. Here we use a notation where $|x\rangle_{\hat{O}}$ is an eigenstate of $\hat{O}$ with eigenvalue $x$. We have analogous expressions in the dual basis: $\left|{ }_{L}\right\rangle=\sum_{j}|2 j \sqrt{\pi}\rangle_{\hat{P}}$, $\left|-_{L}\right\rangle=\sum_{j}|(2 j+1) \sqrt{\pi}\rangle_{\hat{P}}$.

An alternative expression for the codewords can be found by noting that the state $\left|0_{L}\right\rangle \propto \sum_{k, l=-\infty}^{\infty} \hat{S}_{X}^{k} \bar{Z}^{l}|0\rangle$, is a simultaneous +1 eigenstate of the two stabilizer generators $\hat{S}_{X}, \hat{S}_{Z}$ and logical $\bar{Z}$ (in fact, the vacuum state $|0\rangle$ can be replaced by an arbitrary state $|\psi\rangle$ with nonzero overlap with $\left|0_{L}\right\rangle$ in this expression). With the help of Eq. (1), it follows that the logical states can be written

$$
\begin{aligned}
& \left|0_{L}\right\rangle \propto \sum_{k, l=-\infty}^{\infty} e^{-i \pi k l}|2 k \alpha+l \beta\rangle, \\
& \left|1_{L}\right\rangle \propto \sum_{k, l=-\infty}^{\infty} e^{-i \pi(k l+l / 2)}|(2 k+1) \alpha+l \beta\rangle,
\end{aligned}
$$

where the kets on the right-hand side are coherent states $|\zeta\rangle=\hat{D}(\zeta)|0\rangle$. Analogous expressions can be found for the \pm 1 eigenstates of $\bar{X}$ following the same approach.

Since any pair $\alpha, \beta$ that satisfy Eq. (2) is a valid choice, there is an infinity of different GKP codes. The three most common choices are square, rectangular, and hexagonal codes, defined, respectively, by

$$
\begin{aligned}
\text { square: } \alpha & =\sqrt{\frac{\pi}{2}}, \quad \beta=i \sqrt{\frac{\pi}{2}}, \\
\text { rect: } \alpha & =\lambda \sqrt{\frac{\pi}{2}}, \quad \beta=\frac{i}{\lambda} \sqrt{\frac{\pi}{2}}, \quad \lambda>0, \\
\text { hex: } \alpha & =\sqrt{\frac{\pi}{\sqrt{3}}}, \quad \beta=e^{2 i \pi / 3} \sqrt{\frac{\pi}{\sqrt{3}}} .
\end{aligned}
$$

Note that for the square code, the generalized quadratures introduced above are just the usual position and
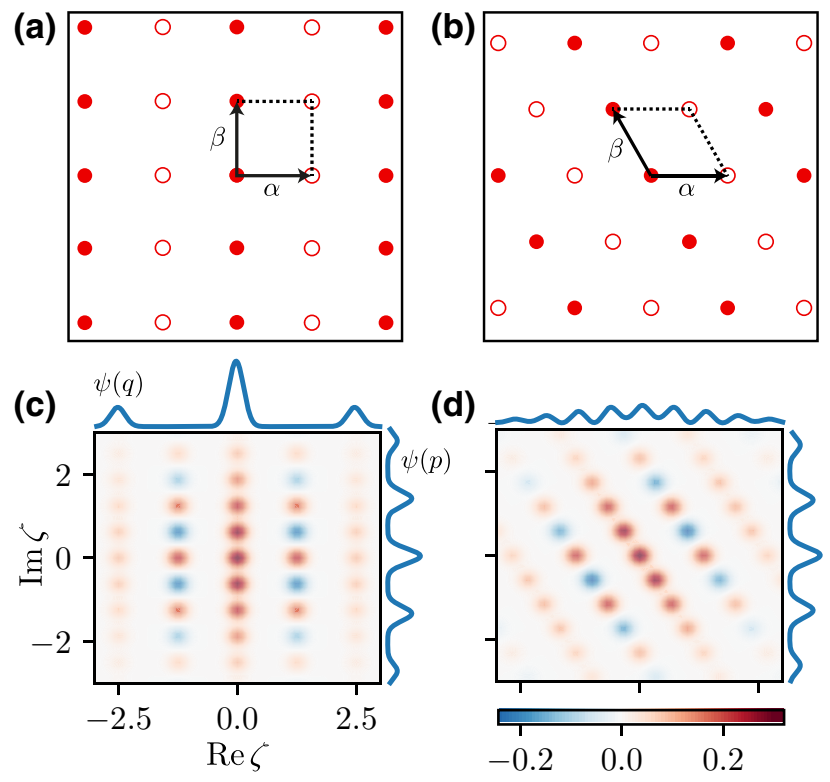

FIG. 1. Square (a) and hexagonal (b) GKP lattices (adapted from Ref. [29]). In Eq. (6) coherent states are placed on the filled (empty) circles for the $\left|0_{L}\right\rangle\left(\left|1_{L}\right\rangle\right)$ state. The area of the lattice parallelogram is $\pi / 2$, which ensures the correct anticommutation relation for the logical operators defined in Eq. (3). Wigner functions $W(\zeta)$ for approximate $\left|\tilde{0}_{L}\right\rangle$ states with $\Delta=0.3$ are shown for square (c) and hexagonal (d) GKP codes. Position $\left[\hat{q}=\left(\hat{a}^{\dagger}+\hat{a}\right) / \sqrt{2}\right]$ and momentum $\left[\hat{p}=i\left(\hat{a}^{\dagger}-\hat{a}\right) / \sqrt{2}\right]$ wave functions are shown in blue.

momentum quadratures $\hat{Q}_{\square}=\hat{q}=\left(\hat{a}+\hat{a}^{\dagger}\right) / \sqrt{2}, \hat{P}_{\square}=$ $\hat{p}=-i\left(\hat{a}-\hat{a}^{\dagger}\right) / \sqrt{2}$. The square and hexagonal GKP lattices are illustrated in Fig. 1.

\section{B. Approximate GKP codewords}

The GKP codespace is a rather abstract construction. The codewords in Eq. (5) are non-normalizable, and in general there is no physical process that can prepare a state lying entirely in the GKP codespace. In practice we have to make do with some type of approximation to Eqs. (5) and (6). Colloquially, we refer to any pair of normalized states $\left|\tilde{\mu}_{L}\right\rangle, \mu=0,1$ that satisfy $\hat{S}_{P}\left|\tilde{\mu}_{L}\right\rangle \rightarrow\left|\tilde{\mu}_{L}\right\rangle$ and $\bar{Z}\left|\tilde{\mu}_{L}\right\rangle \rightarrow(-1)^{\mu}\left|\tilde{\mu}_{L}\right\rangle$ for $P=X, Z$, in some meaningful limit, as an approximate GKP code.

One natural way to define such an approximate code is [30]

$$
\left|\tilde{\mu}_{L}\right\rangle \propto e^{-\Delta^{2} \hat{a}^{\dagger} \hat{a}}\left|\mu_{L}\right\rangle
$$

where we ignore normalization constants, for simplicity. The ideal limit corresponds to $\Delta \rightarrow 0$. Equation (8) introduces a Gaussian envelope over the infinite sums in Eq. (6), such that each coherent state $|\zeta\rangle$ is replaced by $e^{-(1 / 2)\left(1-e^{-2 \Delta^{2}}\right)|\zeta|^{2}}\left|e^{-\Delta^{2}} \zeta\right\rangle \simeq e^{-\Delta^{2}|\zeta|^{2}}\left|e^{-\Delta^{2}} \zeta\right\rangle$, 
which ensures the normalizability of the codewords. The $\left|\tilde{0}_{L}\right\rangle$ codewords for square and hexagonal GKP codes with $\Delta=0.3$ are shown in Fig. 1. It is also possible to view the code defined in Eq. (8) as resulting from a modification of the stabilizers defining the codespace. More precisely, the codewords in Eq. (8) are exact +1 eigenstates of the two commuting nonunitary operators

$$
S_{X, Z}^{\Delta}=e^{-\Delta^{2} \hat{a}^{\dagger} \hat{a}} S_{X, Z} e^{\Delta^{2} \hat{a}^{\dagger} \hat{a}},
$$

with logical operators defined analogously, $\bar{P}^{\Delta}=$

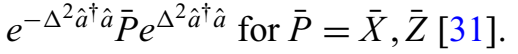

Another common approximate form is found by applying weighted displacements to the ideal codewords

$$
\left|\tilde{\mu}_{L}\right\rangle=\int_{-\infty}^{\infty} d u d v \eta_{\Delta}(u, v) e^{i u v / 2} e^{-i u \hat{P}} e^{i v \hat{Q}_{\mid}}\left|\mu_{L}\right\rangle,
$$

where $\eta_{\Delta}(u, v)$ is concentrated around zero as $\Delta \rightarrow 0$. Equation (8) is recovered with $\eta_{\Delta}(x, y)=$ $e^{-\left(x^{2}+y^{2}\right) /\left(4 \tanh \left(\Delta^{2} / 2\right)\right.} /\left[\pi\left(1-e^{-\Delta^{2}}\right)\right] \simeq e^{-\left(x^{2}+y^{2}\right) / 2 \Delta^{2}} / \pi \Delta^{2}$ [31]. We can use Eq. (5) in Eq. (10) and perform the integral over $v$ to find yet another approximation [1]

$$
\begin{aligned}
\left|\tilde{\mu}_{L}\right\rangle \simeq & \frac{1}{\sqrt{N_{\mu}}} \sum_{j=-\infty}^{\infty} e^{-\Delta^{2} \pi(2 j+\mu)^{2} / 2} \\
& \times \int_{-\infty}^{\infty} d u e^{-\left(u^{2} / 2 \Delta^{2}\right)}|(2 j+\mu) \sqrt{\pi}+u\rangle_{\hat{Q}}
\end{aligned}
$$

where $N_{\mu}=\sqrt{\pi} / 2+\mathcal{O}\left(e^{-\pi / \Delta^{2}}\right)$ as $\Delta \rightarrow 0$. This form has the physical interpretation of a comb of squeezed states with an overall Gaussian envelope.

It is convenient to introduce a metric to quantify how close an arbitrary state $\hat{\rho}$ is to an ideal GKP state. To this end, we introduce a modular "squeezing" parameter for each of the two stabilizers $\hat{S}_{X, Z}$ [32]

$$
\begin{aligned}
& \Delta_{X}=\frac{1}{2|\alpha|} \sqrt{-\log \left(\left|\operatorname{tr}\left[\hat{S}_{X} \hat{\rho}\right]\right|^{2}\right)}, \\
& \Delta_{Z}=\frac{1}{2|\beta|} \sqrt{-\log \left(\left|\operatorname{tr}\left[\hat{S}_{Z} \hat{\rho}\right]\right|^{2}\right)} .
\end{aligned}
$$

The squeezing parameters satisfy $\Delta_{X, Z} \geq 0$, and are zero if and only if the state is an eigenstate of the corresponding stabilizer. For the approximate GKP codewords introduced above, we have $\Delta_{X, Z}=\Delta$ as $\Delta \rightarrow 0$. It is also conventional to measure the modular squeezing in $\mathrm{dB}$

$$
\mathcal{S}_{X, Z}=-10 \log _{10}\left(\Delta_{X, Z}^{2}\right)
$$

For example, the square (hexagonal) approximate codeword shown in Fig. 1 has $\mathcal{S}_{X}=\mathcal{S}_{Z}=10.1 \mathrm{~dB}(9.48 \mathrm{~dB})$, corresponding to an average photon number of approximately $\langle\hat{n}\rangle=4.6$. We from now on drop the subscripts $X, Z$ and simply write $\Delta$ and $\mathcal{S}$ when the two quadratures are approximately equally squeezed and the distinction is unimportant.

\section{Error correcting properties}

The purpose of encoding a logical qubit in a GKP code is that it provides protection from noise through error correction. To see this, consider first an error model consisting of small displacements applied to the oscillator. For an arbitrary displacement $\hat{D}(\zeta)$ we can write $\zeta=(u \alpha+v \beta) / \sqrt{\pi}$, where $u, v$ are real, and consequently

$$
\hat{D}(\zeta)=e^{i u v / 2} e^{-i u \hat{P}} e^{i v \hat{Q}}
$$

From Eq. (5) it is clear that the quantum error correction criteria [33] are formally satisfied for the ideal GKP code for the set of displacement errors $\hat{D}(\zeta)$ such that $|u|,|v|<$ $\sqrt{\pi} / 2$.

This also gives us some insight into why the approximate GKP codewords introduced in the previous section are "good" approximations. As long as $\eta_{\Delta}(u, v)$ in Eq. (10) is sufficiently localized around zero, the "error" introduced in the approximate codewords is small, and as long as we ensure that all the logical operations used in our quantum computation do not amplify these errors too badly, i.e., they are "fault tolerant," then we can expect to perform quantum computation with these approximate GKP codewords with high accuracy (see Sec. II D4 for a more precise discussion around what we mean by not "too badly").

For physical GKP codes and realistic error models, we expect that the error correction criteria are at best only approximately satisfied. Realistic error models for oscillators typically include loss, heating, dephasing, unitary errors due to imperfect implementation of control Hamiltonians, etc. Since the displacement operators form an operator basis, any single-mode noise channel can be expanded in terms of displacements

$$
\mathcal{E}(\hat{\rho})=\int d^{2} \zeta d^{2} \zeta^{\prime} f\left(\zeta, \zeta^{\prime}\right) \hat{D}(\zeta) \hat{\rho} \hat{D}^{\dagger}\left(\zeta^{\prime}\right)
$$

Again, as long as $f\left(\zeta, \zeta^{\prime}\right)$ is sufficiently concentrated around zero, it is in principle possible to remove the noise with high fidelity. Realistic error models, however, typically have some finite support on displacements larger than $\sqrt{\pi} / 2$, which means that the error can not be corrected perfectly, even in the limit $\Delta \rightarrow 0$.

In Fig. 2 we illustrate how the quantum-error-correction properties of the GKP code manifest for a practically relevant noise channel consisting of simultaneous loss and dephasing. More precisely, the noise model is given by the 

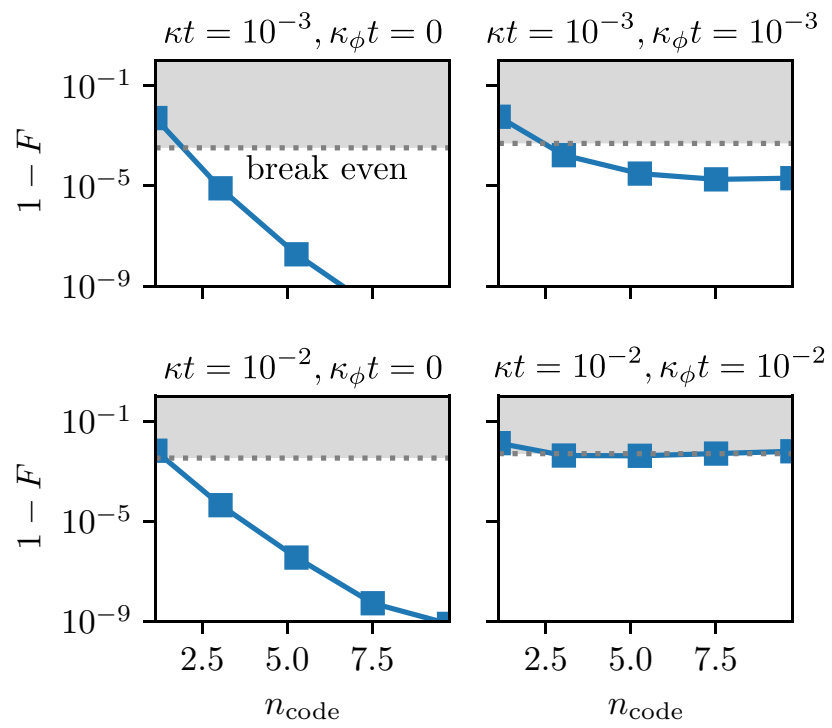

FIG. 2. Average gate fidelity for an approximate square GKP code as a function of photon number $n_{\text {code }}=\left(\left\langle 0_{L}|\hat{n}| 0_{L}\right\rangle+\right.$ $\left.\left\langle 1_{L}|\hat{n}| 1_{L}\right\rangle\right) / 2$, for simultaneous loss and dephasing quantified by $\kappa t$ and $\kappa_{\phi} t$, followed by optimal error correction. The GKP code outperforms the "trivial encoding" in Fock states $|0\rangle$ and $|1\rangle$ whenever the fidelity dips below the gray shaded region.

solution to a Lindblad master equation

$$
\dot{\hat{\rho}}=\kappa \mathcal{D}[\hat{a}] \hat{\rho}+\kappa_{\phi} \mathcal{D}[\hat{n}] \hat{\rho}
$$

with $\mathcal{D}[\hat{A}] \hat{\rho}=\hat{A} \hat{\rho} \hat{A}^{\dagger}-\frac{1}{2} \hat{A} \dagger \hat{A} \hat{\rho}-\frac{1}{2} \hat{\rho} \hat{A}^{\dagger} \hat{A}$, integrated up to a fixed time $t$. The noise strength in this model is thus characterized by two dimensionless numbers, $\kappa t$ and $\kappa_{\phi} t$, describing pure loss and dephasing, respectively. The noise is then followed by the optimal recovery channel that maximizes the average gate fidelity [34] with the identity channel. This optimal error-correction map can be found numerically [35], but does not represent a practical errorcorrection procedure - it merely puts an upper bound on the fidelity that can be achieved and illustrates the intrinsic error-correction properties of the code.

The results in Fig. 2 show that the GKP code has an excellent potential to correct loss errors [35], but is rather poor against dephasing (there are other bosonic codes that perform far better against this latter type of noise [29]). The sensitivity to dephasing is not surprising, as a rotation of phase space by a small angle gives a large displacement for large amplitudes. In practice, dephasing might arise not only due to the intrinsic frequency fluctuations of the oscillator used to encode the GKP code (which can be made very small), but also due to off-resonant coupling to ancilliary quantum systems used to control the oscillator [3-5]. It is therefore crucial to minimize such residual couplings in practical implementations of GKP codes. A similar issue is likely to arise if there are over-rotations and/or unwanted residual Hamiltonian terms due to miscalibrated unitary gates, and very precise quantum control is therefore important for GKP codes.

\section{Logical operations on GKP codes}

One of the attractive properties of GKP codes is that, apart from state preparation, all logical Clifford operations can be performed using only Gaussian operations, that is, interactions that are at most quadratic in creation and annihilation operators and homodyne measurements on the oscillator. In this section we describe how to perform logical Pauli measurements and unitary Clifford gates, leaving the more difficult topic of state preparation and error correction to Sec. III.

\section{Pauli quadrature measurements}

Destructive logical measurements in any Pauli basis $\left(\mathcal{M}_{X, Y, Z}\right)$ can be performed by measuring one of three respective quadratures

$$
\begin{aligned}
& \mathcal{M}_{X}: \text { measure }-\hat{P}, \\
& \mathcal{M}_{Y}: \text { measure } \hat{Q}-\hat{P}, \\
& \mathcal{M}_{Z}: \text { measure } \hat{Q},
\end{aligned}
$$

and rounding the outcome to the nearest multiple of $\sqrt{\pi}$. If the result is an even multiple, report a +1 outcome, and if the result is an odd multiple, report -1 . That this gives a logical Pauli measurement follows from Eq. (4). An attractive feature of this measurement scheme is that it is robust to small displacement errors - precisely the type of errors the GKP code is meant to be robust against - and can in this sense be said to be fault tolerant. The procedure is illustrated for an $\mathcal{M}_{Z}$ measurement on an approximate square GKP code in Fig. 3(a).

Quadrature measurements are routinely performed in both the microwave and optical domain. Performing such measurements on an encoded GKP qubit is, however, not as straight forward. In the context of cQED and other approaches where the GKP state is encoded in a localized high-quality mode, such as the standing modes of a cavity, the ability to rapidly perform quadrature measurements contradicts the requirement of the oscillator mode to be long lived. It is therefore necessary to either tune the oscillator decay rate $\kappa$ from a small to a large value prior to measurement, or to map the encoded information from a high- $Q$ to a low- $Q$ mode (with $Q \sim 1 / \kappa$ the quality factor) [36].

The situation is further complicated by the fact that the measurement efficiency for homodyne detection is limited in practice. The ability to distinguish the codewords deteriorates rapidly with decreasing measurement efficiency, as shown in Fig. 3(b). A measurement efficiency $\eta$ below unity means that the GKP state shrinks towards vacuum, 


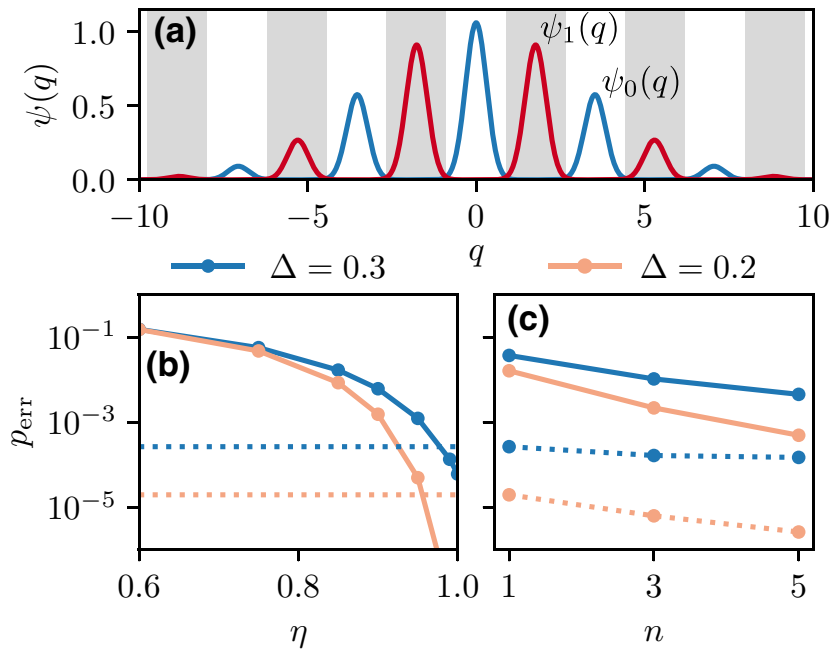

FIG. 3. (a) Position wave functions for logical zero (blue) and one (red) for a square GKP code with $\Delta=0.3$. A logical $\mathcal{M}_{Z}$ measurement can be executed by first measuring position (homodyne measurement) and binning the result. The white (gray) regions correspond to a logical zero (one) outcome. (b) Probability of mistaking logical zero for one under a homodyne measurement with measurement efficiency $\eta$ for $\Delta=0.3$ $(\mathcal{S}=10.1 \mathrm{~dB}$, blue solid) and $\Delta=0.2(\mathcal{S}=13.8 \mathrm{~dB}$, orange solid). Note that the bin boundaries are scaled by $\sqrt{\eta}$ to account for measurement inefficiency. For comparison, the dotted lines show the corresponding measurement error with one round of noiseless phase estimation using the scheme in Fig. 4(b). (c) Same as (b) but for a majority vote over $n$ rounds of noiseless phase estimation using the schemes in Figs. 4(a) (solid) and 4(b) (dotted).

and it is important to compensate for this (assuming that $\eta$ itself is known) by rescaling the measurement bins [white and pink in Fig. 3(a)] appropriately. More precisely, we now round to the nearest integer multiple of $\sqrt{\eta \pi}$ [37]. To produce the numerical results in Fig. 3(b) we take an approximate $\left|\tilde{0}_{L}\right\rangle$ state, apply a pure loss channel with $\eta=e^{-\kappa t}$, followed by an ideal measurement of the position quadrature and bin the result.

In the microwave domain, state-of-the-art measurement efficiencies are well below $90 \%$, even with the use of near quantum-limited amplifiers [38,39]. The results in Fig. 3(b) show that measurement efficiencies will have to be improved for this approach to be promising for distinguishing GKP codewords with high fidelity. For example, at $\eta=75 \%$ - a high but not unreasonable value for a microwave measurement chain - the error probability is about $p_{\text {err }} \simeq 5.6 \%(4.1 \%)$ for $\Delta=0.3(0.2)$. For comparison, at $\eta=90 \%$ we find $0.61 \%(0.15 \%)$. Note that rather large measurement efficiencies are required to have a substantial benefit from lowering $\Delta$.

The measurement efficiency may be improved if we can amplify the quadrature information prior to releasing the GKP state to a standard microwave measurement chain [40]. Although most theoretical work on GKP codes assume high-efficiency quadrature measurements [22-25], it is an open question whether the stringent demands required for scalable, fault-tolerant quantum computing can be met with this approach. We return to this question in Sec. IV where we discuss concatenation with topological codes.

\section{Pauli phase estimation}

An alternative, and nondestructive, way to do logical Pauli measurements is by performing phase estimation using an ancillary system. In the simplest case this task can be performed using a discrete two-level system as an ancilla, removing the need to perform direct quadrature measurements on an encoded GKP state.

Since the logical GKP Paulis are unitary displacement operators, $\bar{X}=\hat{D}(\alpha), \bar{Z}=\hat{D}(\beta)$, their eigenvalues are of the form $e^{i \theta}$ with $\theta \in[0,2 \pi)$. The task of estimating an eigenvalue of a unitary operator, or equivalently the "phase" $\theta$, is generally known as phase estimation. A variety of different phase-estimation protocols exist, with trade-offs in terms of efficiency and complexity $[31,41,42]$. We here focus on a simple nonadaptive scheme using a single two-level ancilla. Specifically we consider the scheme that was used in the experiments in Refs. [2,3], illustrated in Fig. 4(a), as well as a modified version that has theoretically been shown to give better performance, shown in Fig. 4(b) $[31,43]$.

The central component of these schemes is a controlleddisplacement gate $C \hat{D}(\zeta)$, which applies a displacement on the GKP mode $\hat{D}( \pm \zeta / 2)$ conditioned on the state of the two-level ancilla. See Fig. 9 for possible implementations of this gate in cQED. At the end of the circuit, the ancilla is

(a)

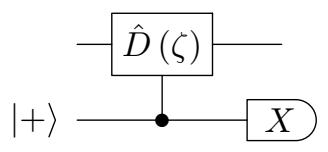

(b)

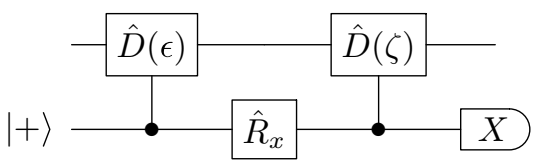

FIG. 4. (a) One round of phase estimation. The controlled displacement is defined as $C \hat{D}(\zeta)=\hat{D}(\zeta / 2) \otimes\left|0_{a}\right\rangle\left\langle 0_{a}\right\rangle+$ $\hat{D}(-\zeta / 2) \otimes\left|1_{a}\right\rangle\left\langle 1_{a}\right\rangle$, with $\left|0_{a} / 1_{a}\right\rangle$ the state of the ancilla. For $\bar{X}$ measurement $\zeta=\alpha$ and for $\bar{Z}$ measurement $\zeta=\beta$. (b) Improved phase-estimation scheme. Here, $\epsilon$ is a small displacement orthogonal to $\zeta$, i.e., $\arg \epsilon=\arg \zeta+\pi / 2$. For $\bar{X}$ measurement we set $\hat{D}(\epsilon / 2)=e^{-i \lambda \hat{Q}}$ and for $\bar{Z}$ measurement $\hat{D}(\epsilon / 2)=e^{i \lambda \hat{P}}$, with $\lambda \in \mathbb{R}$. The $\hat{R}_{x}$ gate is defined as $\hat{R}_{x}=e^{-i \pi \hat{\sigma}_{x} / 4}$. Note that with this definition of the controlled displacement gate, the state is displaced by $\zeta / 2$ from the codespace after the measurement. This can be corrected for with another displacement if necessary. 
measured in the $X$ basis. Consider first the simplest scheme in Fig. 4(a). The probability of getting an $X= \pm$ outcome for the ancilla measurement is given by

$$
P( \pm)=\frac{1}{2}\left[1 \pm \frac{1}{2}\left(\langle\hat{D}(\zeta)\rangle+\left\langle\hat{D}^{\dagger}(\zeta)\right\rangle\right)\right] .
$$

To be concrete, let us take $\hat{D}(\zeta)=\bar{Z}$. For an ideal GKP code we have $\left\langle\mu_{L}|\bar{Z}| \mu_{L}\right\rangle= \pm 1$ for $\mu=0,1$, and thus $P(+)=1, P(-)=0$ for the $\left|0_{L}\right\rangle$ state. For an approximate $\left|\tilde{0}_{L}\right\rangle$ state, on the other hand, there is a nonzero probability of getting a -1 outcome. Using Eq. (11) one can show that $\left\langle\tilde{0}_{L}|\bar{Z}| \tilde{0}_{L}\right\rangle \simeq e^{-\pi \Delta^{2} / 4}$ for small $\Delta$, such that the measurement error becomes

$$
p_{\text {err }}=P(-) \simeq \frac{1}{2}\left(1-e^{-\pi \Delta^{2} / 4}\right) \simeq \frac{\pi \Delta^{2}}{8} .
$$

Interestingly, the performance can be improved significantly using the scheme shown in Fig. 4(b). Here, a small controlled displacement orthogonal to the logical displacement is performed first. In the case of a $\bar{Z}=e^{i \sqrt{\pi} \hat{Q}}$ measurement we set $\hat{D}(\epsilon / 2)=e^{i \lambda \hat{P}}$ with $\lambda$ real. The intuition behind the scheme is that this gives a better approximation to a measurement of the approximate logical Pauli operator $\bar{Z}^{\Delta}$ introduced in Sec. II B [31]. In this case, one can show that the measurement error becomes

$$
p_{\text {err }} \simeq \frac{1}{2}\left\{1-e^{-\left(\pi \Delta^{2} / 4\right)}\left[e^{-\left(\lambda^{2} / \Delta^{2}\right)}+\sin (\sqrt{\pi} \lambda)\right]\right\} .
$$

We can treat $\lambda$ as a free parameter to be optimized. In the small $\Delta$ limit $p_{\text {err }}$ is minimized for $\lambda \simeq \sqrt{\pi} \Delta^{2} / 2$ in which case one finds $p_{\text {err }} \simeq 0.4 \Delta^{6}$, a significant improvement over Eq. (19) [43].

In Fig. 3(c) we show $p_{\text {err }}$ for a majority vote over $n$ rounds of ideal phase estimation with a noiseless twolevel ancilla for the two respective schemes. For the simple scheme in Fig. 4(a) and $\Delta=0.3(0.2)$ we find $p_{\text {err }} \simeq 3.7 \%$, $1.0 \%$, and $0.4 \%(1.6 \%, 0.2 \%$, and $0.05 \%)$ for $n=1,3$, and 5 measurements, respectively. For the improved scheme in Fig. 4(b) we find $p_{\text {err }} \simeq 2.6 \times 10^{-4}, 1.6 \times 10^{-4}$, and $1.5 \times 10^{-4}\left(1.9 \times 10^{-5}, 6.2 \times 10^{-6}\right.$, and $\left.2.5 \times 10^{-6}\right)$ for the same parameters. These results are produced by numerically computing the probability of a -1 measurement outcome on a $\left|\tilde{0}_{L}\right\rangle$ state for the circuits in Fig. 4, and in the case of the scheme in (b) optimizing over the parameter $\lambda$.

These results show that phase estimation, modified to better distinguish the approximate Pauli operators of a physical GKP code, can lead to very small measurement errors when the ancilla is noiseless. In Sec. III we discuss how this approach can form the basis for a statepreparation scheme, by measuring stabilizer operators in place of logical Paulis. A fundamental obstacle to this approach, however, is that errors on the ancilla qubit can propagate back to the GKP mode. In particular, a bit flip of the ancilla qubit during the controlled displacement gate $C \hat{D}(\zeta)$ leads to a large, random displacement of the GKP code. In Sec. III B we discuss a potential way to make these schemes robust to such ancilla errors.

\section{Clifford gates and Clifford frames}

As already mentioned, Clifford gates can be performed using interactions that are at most quadratic in the creation and annihilation operators. Specifically, the Clifford group can be generated, for example, from the Hadamard $(H)$, phase $(S)$, and CNOT $\left(C_{X}\right)$ gates [1]

$$
\bar{H}=e^{(i \pi / 4)\left(\hat{Q}^{2}+\hat{P}^{2}\right)}, \quad \bar{S}=e^{(i / 2) \hat{Q}^{2}}, \quad \bar{C}_{X}=e^{-i \hat{Q} \otimes \hat{P}},
$$

where we use the generalized (code-dependent) quadratures $\hat{Q}, \hat{P}$ introduced in Sec. II A. For the $\bar{C}_{X}$ gate the first mode is the control and the second mode the target. Together with logical basis measurements $\mathcal{M}_{Z}$ and preparation of encoded states $\left|0_{L}\right\rangle$ and $\left|A_{L}\right\rangle=\frac{1}{\sqrt{2}}\left(\left|0_{L}\right\rangle+\right.$ $\left.e^{i \pi / 4}\left|1_{L}\right\rangle\right)$, this forms a universal set.

It should be emphasized, however, that the gates in Eq. (21) are in general only approximate logical gates on approximate GKP codes, and, despite being unitary operations, may reduce the quality of the encoded information by making the codewords harder to distinguish [this can be seen from the fact that the gates do not commute with the envelope operator introduced in Eq. (8)] [21,37].

Due to the approximate nature of the logical gates on physical GKP codewords, it is desirable to minimize the number of Clifford gates in a given quantum circuit. An elegant solution to this problem is to make use of a so-called Clifford frame, where single-qubit Cliffords are tracked "in software" $[44,45]$. The idea of the (singlequbit) Clifford frame is as follows: An arbitrary quantum circuit $\mathcal{C}$, written in terms of preparation of $\left|0_{L}\right\rangle$ and $\left|A_{L}\right\rangle$ states, gates from the set $\left\{H, S, C_{X}\right\}$, and Pauli measurements $\mathcal{M}_{Z}$, can be replaced by an equivalent circuit $\mathcal{C}^{\prime}$, where the state preparation is identical, the measurements are in any Pauli basis, and all the gates are from the set

$$
C_{\sigma_{i} \sigma_{j}}=I \otimes I-\frac{1}{2}\left(I-\sigma_{i}\right) \otimes\left(I-\sigma_{j}\right),
$$

where $\sigma_{i, j} \in\{X, Y, Z\}$ runs over the usual Pauli operators. Moreover, the number of qubits, two-qubit gates, and measurements in the new circuit $\mathcal{C}^{\prime}$ is the same as in $\mathcal{C}$, while all single-qubit gates have been removed. Constructing $\mathcal{C}^{\prime}$ from $\mathcal{C}$ is straight forward. One simply commutes the $H$ and $S$ gates through all the $C_{X}$ gates, mapping them to new gates from the set Eq. (22) (and by-product single-qubit Pauli gates) in the process, and finally absorb any singlequbit Cliffords and Paulis into the measurements, mapping $\mathcal{M}_{Z}$ to general Pauli measurements [46]. An example is given in Fig. 5. 
(a)

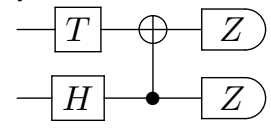

(c) $Z_{1}=+1$

$|A\rangle$

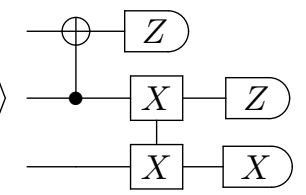

(b)

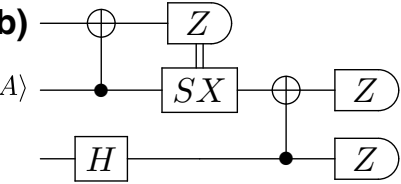

(d) $Z_{1}=-1$ $\frac{\sigma_{i}}{\sigma_{j}}=\hat{C}_{\sigma_{i}, \sigma_{j}}$

$|A\rangle$

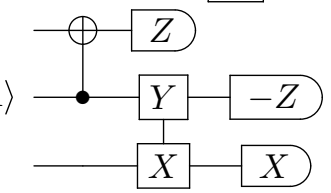

FIG. 5. Example computation in the (single-qubit) Clifford frame. A general quantum circuit (a) can be rewritten using only (adaptive) Clifford gates and magic states $|A\rangle=T|+\rangle$, with $T=\operatorname{diag}\left(1, e^{i \pi / 4}\right)(\mathrm{b})$. The circuit in (b) can moreover be rewritten as either (c) or (d), depending on the measurement outcome $Z_{1}= \pm 1$ of the first qubit. The generalized control gates are defined in Eq. (22). For GKP qubits, updating the Clifford frame amounts to changing the phase of local oscillators (c.f. Fig. 6) so that single-qubit Clifford gates are done in software.

For GKP-encoded qubits, the gate set Eq. (22) is particularly attractive, because switching between gates in this set is essentially a "free" operation in many physical platforms. More precisely, an encoded version of the gateset for GKP codes is realized by [37]

$$
\bar{C}_{\sigma_{i} \sigma_{j}}=e^{i \hat{s}_{i} \otimes \hat{s}_{j}},
$$

where $\hat{s}_{1}=-\hat{P}, \hat{s}_{2}=\hat{Q}-\hat{P}, \hat{s}_{3}=\hat{Q}$ are the three quadratures corresponding to logical $\bar{X}, \bar{Y}$, and $\bar{Z}$, respectively.

Any gate from the set Eq. (23) can be generated from an interaction of the form $\hat{H}_{\theta, \phi} \propto e^{i \theta} \hat{a} \hat{b}^{\dagger}+e^{i \phi} \hat{a} \hat{b}+$ H.c., where $\hat{a}$ and $\hat{b}$ are annihilation operators for the two respective modes. In turn, $\hat{H}_{\theta, \phi}$ can be realized, for example, from a three-wave mixing interaction with a classical pump with two pump-tone frequencies at the sum and difference of the two GKP modes, respectively, and $\theta$ and $\phi$ set by the two corresponding pump phases, see Fig. 6(a). Alternatively, it can be realized from a four-wave mixing interaction, using four pump tones, as shown in Fig. 6(b). Updating the Clifford frame thus simply amounts to updating the programming of classical pump phases. Albeit logical gates between two GKP qubits have not yet been demonstrated at the time of writing, the ability to engineer interactions of the form $\hat{H}_{\theta, \phi}$ have already been used for other applications in cQED [36,47-50].

\section{Error spread through gates}

An important consequence of the fact that Clifford gates on GKP codes can be generated by quadratic Hamiltonians is that this guarantees that errors are not amplified

(a)

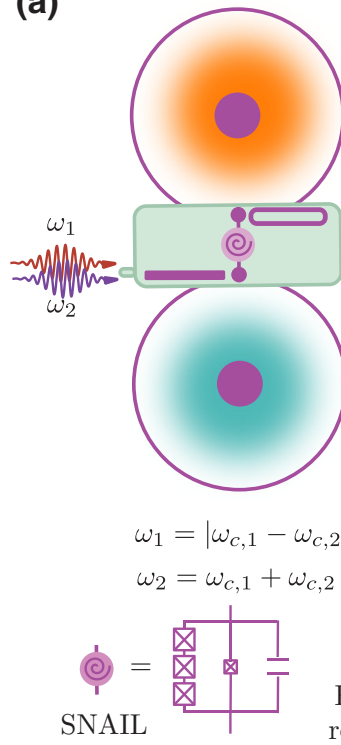

(b)

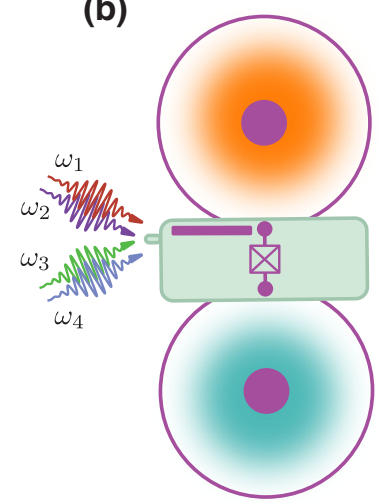

$$
\left|\omega_{1}-\omega_{2}\right|=\left|\omega_{c, 1}-\omega_{c, 2}\right|
$$$$
\omega_{3}+\omega_{4}=\omega_{c, 1}+\omega_{c, 2}
$$

Readout
resonator

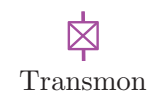

FIG. 6. Illustration of how the Hamiltonian $\hat{H}_{\theta, \phi} \propto e^{i \theta} \hat{a} \hat{b}^{\dagger}+$ $e^{i \phi} \hat{a} \hat{b}+$ H.c., required for realization of the two-qubit Clifford gates $\bar{C}_{\sigma_{i} \sigma_{j}}$ in Eq. (23), may be realized in a cQED architecture. The two GKP resonators, shown in orange and blue, have frequencies $\omega_{c, 1}$ and $\omega_{c, 2}$, respectively. In (a), a superconducting nonlinear asymmetric inductive element (SNAIL) is used for the coupling [51]. At an appropriate flux bias, the SNAIL exhibits a strong third-order nonlinearity. Two microwave drives are applied at frequencies $\omega_{1}$ and $\omega_{2}$ so that $\omega_{1}=\left|\omega_{c, 1}-\omega_{c, 2}\right|$ and $\omega_{2}=\omega_{c, 1}+\omega_{c, 2}$. Because of the third-order nonlinearity, a single photon at $\omega_{1}$ is consumed to convert a photon at $\omega_{c, 1}$ to that at $\omega_{c, 2}$. This leads to an interaction of the form $\hat{a}^{\dagger} \hat{b} e^{i \theta}+$ H.c., where the strength of the interaction and $\theta$ depend on the strength of the microwave drive at $\omega_{1}$ and its phase, respectively. Similarly, a single photon at $\omega_{2}$ is consumed to create two photons, one at $\omega_{c, 1}$ and the other at $\omega_{c, 2}$. This leads to an interaction of the form $\hat{a}^{\dagger} \hat{b}^{\dagger} e^{i \phi}+$ H.c., where, again, the strength of the interaction and $\phi$ is set by the drive strength and phase, respectively. Alternatively, it is possible to engineer $\hat{H}_{\theta, \phi}$ using a transmon as shown in (b). In this case, four pump tones can be used to generate the interaction. Due to the fourth-order nonlinearity of the transmon, a photon each from the pumps at $\omega_{3}$ and $\omega_{4}$ such that $\omega_{3}+\omega_{4}=\omega_{c, 1}+\omega_{c, 2}$, are consumed to generate two photons at $\omega_{c, 1}$ and $\omega_{c, 2}$. On the other hand, photons from the pumps at $\omega_{1}$ and $\omega_{2}$ such that $\left|\omega_{1}-\omega_{2}\right|=\left|\omega_{c, 1}-\omega_{c, 2}\right|$, convert a photon at $\omega_{c, 1}$ to $\omega_{c, 2}$ via four-wave mixing.

in a bad way by the gates. Consider, for example, the $\bar{C}_{X} \equiv \bar{C}_{Z X}=e^{-i \hat{Q} \otimes \hat{P}}$ gate from the set Eq. (23) (the other gates in the set behave analogously), and assume that a small displacement error $e^{-i u \hat{P}} e^{i v \hat{Q}}$ [c.f. Eq. (14)] is present on the first (control) mode prior to performing the gate. The factor $e^{i v \hat{Q}}$ commutes with the $\bar{C}_{X}$ gate, but since

$$
\left(e^{-i u \hat{P}} \otimes I\right) e^{-i \hat{Q} \otimes \hat{P}}=e^{-i \hat{Q} \otimes \hat{P}}\left(e^{-i u \hat{P}} \otimes e^{i u \hat{P}}\right)
$$


we see that the $\bar{C}_{X}$ gate spreads a displacement error $e^{i u \hat{P}}$ to the second (target) mode. Even though the error has spread, small displacements spread to small displacements, and the error can be corrected by a subsequent round of error correction. This is exactly analogous to a transversal $\bar{C}_{X}=C_{X}^{\otimes n}$ between two binary code blocks of $n$ qubits, where, say, $t X$ errors on the control block can spread to $t X$ errors on the target block.

In this sense the Clifford gates on GKP codes are fault tolerant. This is, however, only a statement about an ideal implementation of a gate such as Eq. (23). In a realistic implementation, where the quadratic interaction $\hat{H}_{\theta, \phi}$ stems from an underlying nonlinearity (c.f. Fig. 6), there will unavoidably be spurious higher-order terms present as corrections to $\hat{H}_{\theta, \phi}$. Such terms might amplify and spread errors in a bad way, and it is therefore crucial that they are made as small as possible. Again, it is not expected that GKP codes can suppress errors arbitrarily. The goal is to suppress errors to sufficiently low levels that the resource overhead for the next level of protection is reduced, as discussed further in Sec. IV.

\section{STATE PREPARATION AND ERROR CORRECTION}

\section{A. State preparation using two-level ancilla}

One way to prepare a GKP state is to nondestructively measure the stabilizers and a corresponding logical Pauli. For example, a measurement of $\hat{S}_{X}$ and $\bar{Z}$, both with +1 outcomes, would correspond to a preparation of the ideal $\left|0_{L}\right\rangle$ state. We discussed how logical Paulis can be measured using phase estimation in Sec. II D2. These ideas can be extended to measuring the stabilizers $\hat{S}_{X}, \hat{S}_{Z}$, and introducing feedback displacements to steer the state towards the codespace of an approximate GKP code.

Several protocols have been developed to this end [2, $3,10,31,41,42]$. To keep the discussion concrete, we here focus on the scheme illustrated in Fig. 7, which is the scheme used in the experimental demonstrations of GKP codewords in Ref. [3]. Let us first consider in more detail the circuit labeled "Sharpen." This is simply a version of the standard phase-estimation circuit we introduce in Fig. 4(a) with a feedback displacement used to steer the state towards a +1 eigenstate of $\hat{D}(\zeta)$. The probability of getting \pm outcomes here are, respectively, $P_{\pi / 2}( \pm)=$ $\frac{1}{2}(1 \pm \Im\langle\hat{D}(\zeta)\rangle)$. To prepare a state with a target phase value $\theta=0$, we introduce a measurement-dependent displacement of $\hat{D}( \pm \epsilon / 2)$ along a direction orthogonal to $\hat{D}(\zeta)$, so that for a \pm outcome an eigenstate state with eigenvalue $e^{i \theta}$ is mapped to one with $e^{i(\theta \mp \zeta \epsilon)}$. This ensures that $\theta=0(\bmod 2 \pi)$ is a stable fixed point, while $\theta=\pi$ $(\bmod 2 \pi)$ is unstable, for sufficiently small $\epsilon$.

Of course, for a fixed $\epsilon$, we can not prepare a phase arbitrarily close to zero using the above procedure, but

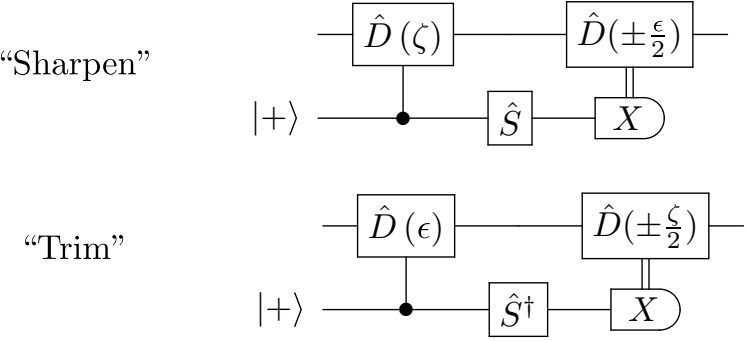

FIG. 7. "Sharpen" and "Trim" protocols used to prepare approximate GKP codestates, the controlled displacement gate is defined as in Fig. 4. The parameter $\epsilon$ is small with $\arg \epsilon=$ $\arg \zeta+\pi / 2$, such that $\hat{D}(\epsilon)$ is a small displacement orthogonal to $\hat{D}(\zeta)$. The $\hat{S}=\operatorname{diag}(1, i)$ gate is the usual phase gate on the ancilla.

this is also not desirable. In practice, the scheme will be limited by experimental imperfections, such as unwanted nonlinearities and dephasing, as the photon number of the state increases. It is therefore better to directly target an approximate GKP state as defined in Sec. II B, where the choice of $\Delta$ should be optimized based on experimental considerations.

It was shown in Ref. [31] that by alternating the phase estimation of $\hat{D}(\zeta)$ ("Sharpen" in Fig. 7) with phase estimation of a small orthogonal displacement $\hat{D}(\epsilon)$ ("Trim" in Fig. 7) one can prepare an approximate GKP state of the form Eq. (8), with $\Delta \sim \sqrt{\epsilon}$. The intuition behind the scheme is that the first step "sharpens the peaks" of the target GKP state by bringing it closer to a +1 eigenstate of $\hat{D}(\zeta)$, while the second step "trims the envelope" of the state by weakly measuring the orthogonal quadrature [31].

To prepare a logical state, say $\left|\tilde{0}_{L}\right\rangle$, one can first alternate many Sharpen-and-Trim cycles of the two stabilizers $\hat{S}_{X}, \hat{S}_{Z}$ to project the state onto the logical subspace. Once in the codespace, a single phase estimation round of $\bar{Z}=$ $\hat{D}(\beta)$ suffices to project onto one of the two logical $Z$ basis states. This can be done using, for example, either of the two circuits in Fig. 4. The full protocol is illustrated in Fig. 8. Alternatively, one can repeat the logical $\bar{Z}$ measurement a few times and postselect on getting identical outcomes, to increase the preparation fidelity [3,31] (see also Fig. 3). Finally, a Pauli correction can be applied if necessary to prepare $\left|\tilde{0}_{L}\right\rangle$.

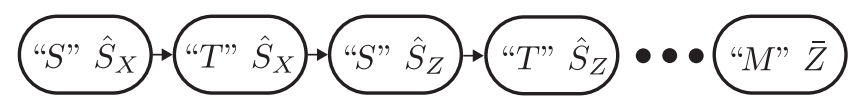

FIG. 8. Preparation of a logical state can be done by alternating "Sharpen" (" $S$ ") and "Trim" (" $T$ ") cycles for the two stabilizers $\hat{S}_{X}, \hat{S}_{Z}$ to project onto the codespace, and finally perform a nondestructive $\bar{Z}$ measurement (" $M$ ") using, e.g., one of the two circuits in Fig. 4. 
Various optimizations of the "Sharpen-Trim" scheme are possible, as well as measurement-free versions. We refer the reader to Refs. [10,31,52] for further details. We also note that optimal control methods have successfully been used to prepare other bosonic codes [4,5], and that similar techniques may prove useful for GKP state preparation as well.

\section{B. Fault tolerance in state preparation}

An issue with the scheme illustrated in Fig. 7 (as well as the Pauli measurement schemes in Fig. 4) is that ancilla errors can propagate to the GKP code and lead to uncorrectable errors. In particular, an ancilla bit flip at a random time during the controlled displacement of the "Sharpen" step leads to a, potentially large, random displacement error. (A bit-flip during the controlled displacement of the "Trim" step is much less serious as it only leads to a displacement error of magnitude $\sim|\epsilon|$.) These simple circuits are, in other words, not fault tolerant to the dominant error channels, such as relaxation, on the ancilla qubit.

It is noteworthy, however, that there is a certain amount of built-in robustness in the phase-estimation circuits, in that phase flips on the ancilla qubit are relatively benign: $Z$ errors on the ancilla commute with the gates and thus only lead to measurement errors. For the "Sharpen" circuit a measurement error only leads to a small displacement of magnitude $|\epsilon / 2|$ in the wrong direction. This will broaden the GKP peaks, but is not very harmful as long as it does not happen too often. For the "Trim" circuit, a measurement error will lead to a large displacement $\hat{D}( \pm \zeta / 2)$ in the wrong direction, however, these displacements are equivalent up to the stabilizer $\hat{D}(\mp \zeta)$, and thus does not lead to a logical error. A measurement error in the final "Measure" step illustrated in Fig. 8 is more serious, as it leads to a logical error. However, as already mentioned, we may repeat this measurement to suppress such measurement errors. In the following, we outline a potential approach to increase the robustness of these circuits by exploiting the natural robustness against phase flips of the phase-estimation protocols.

\section{Biased noise ancilla}

A few different protocols can be applied for more robust phase estimation. For example, the approach considered in Ref. [54] is based on using an extra flag qubit to prevent a single ancilla error from introducing a large displacement error in the GKP state. More hardware-efficient robustness against single-transmon ancilla error can also be achieved by applying the technique known as $\chi$ matching [55]. In this approach, the transmon's $|g\rangle$ and $|f\rangle$ levels are used as computational states and the transmon-cavity coupling is engineered so that the GKP resonator is transparent to a single relaxation error from state $|f\rangle$ to $|e\rangle$ in the transmon.
The approach we outline here for robust phase estimation is based on using a biased-noise ancilla qubit [53].

Biased-noise qubits couple asymmetrically with the environment so that one type of error, such as phase flips or Pauli- $Z$ errors, is more common than others, such as bit flips or Pauli- $X, Y$ errors. In such qubits it is convenient to define a quantity called the bias, which is the ratio of the dominant error and the sum of all other errors $\eta=$ $p_{z} /\left(p_{x}+p_{y}\right)$. For pure- $Z$ noise the bias $\eta=\infty$, while for isotropic or depolarizing noise $\eta=0.5$. Many examples of such biased-noise qubits exists, including the heavy fluxonium qubit [56], the soft $0-\pi$ qubit [57], and the Kerr [49] and dissipative [58] cat qubits. In the trapped-ion implementation of GKP codes [2], the ancillary pseudospin states used to control the motional mode naturally has such a strong bias.

Thanks to the robustness against phase errors, a possible path towards creating a fault-tolerant state-preparation scheme is to use a biased-noise ancilla qubit where bit-flip errors are heavily suppressed [53]. To be able to implement the circuits in Figs. 4 and 7 fault tolerantly, it is however crucial that we can perform the required controlleddisplacement gates while preserving a strong suppression of bit-flip errors. While there are several candidates for strongly biased noise qubits in the superconducting circuit platform [56,57,59], here we focus on the Kerr-cat qubit as an illustrative example, as this provides a particularly straight forward, hardware-efficient, realization of the operations required for the phase-estimation protocols.

In the Kerr-cat qubit the logical states are superpositions of coherent states $| \pm\rangle \propto|\alpha\rangle \pm|-\alpha\rangle(|0 / 1\rangle \simeq| \pm \alpha\rangle)$ of the electromagnetic field stored in a nonlinear oscillator. More precisely, these states are eigenstates of a Kerr-nonlinear oscillator in the presence of a two-photon pump: $\hat{H}_{\text {cat }}=$ $-K \hat{a}^{\dagger 2} \hat{a}^{2}+K \alpha^{2}\left(\hat{a}^{\dagger 2}+\hat{a}^{2}\right)$, where we are working in the rotating frame of the oscillator where the two-photon pump is resonant [60]. The cat states are separated from the closest eigenstates by a gap $\omega_{\text {gap }} \simeq 4 K \alpha^{2}$, and we take $\alpha$ to be real for simplicity. Crucially, realistic noise channels for this system, including photon loss, heating, and dephasing, are highly unlikely to cause transitions between the $|0\rangle$ and $|1\rangle$ states. More precisely, bit flips are exponentially suppressed in $\alpha^{2}$ compared to phase flips leading to an exponentially large bias in $\alpha^{2}$ [60].

Returning to the circuits in Fig. 7, we first note that preparation and measurement in the $X$ basis, as well as the $\hat{S}, \hat{S}^{\dagger}$ phase gates, are bias-preserving operations, i.e., the effective error channel remains biased towards $Z$ errors when performing these operations. These operations have already been demonstrated experimentally for the Kerr-cat qubit [49]. On the other hand, a controlled displacement can be implemented with a beam-splitter interaction

$$
\hat{H}_{C D}=i\left(g \hat{a}_{\mathrm{cat}} \hat{a}_{\mathrm{GKP}}^{\dagger}-g^{*} \hat{a}_{\mathrm{cat}}^{\dagger} \hat{a}_{\mathrm{GKP}}\right),
$$


where the subscript refers to the cat and GKP mode, respectively, and $g$ is an (in general complex) interaction strength. To show that this approximately leads to a conditional displacement, we project the Hamiltonian onto the logical cat subspace $\hat{P}_{\text {cat }}=|\alpha\rangle\langle\alpha|+|-\alpha\rangle\langle-\alpha\rangle$ : $P_{\text {cat }} \hat{H}_{C D} P_{\text {cat }}=i \alpha\left(g \hat{a}_{\mathrm{GKP}}^{\dagger}-g^{*} \hat{a}_{\mathrm{GKP}}\right) \bar{Z}_{\mathrm{cat}}+\mathcal{O}^{*}\left(e^{-2 \alpha^{2}}\right)$, with $\bar{Z}_{\text {cat }}$ the logical Pauli- $Z$ operator for the cat qubit (we use the $\mathcal{O}^{*}[f(x)]$ notation to suppress polynomial factors in $x$ [61]). Evolution under this interaction for a time $t$ thus leads to a controlled displacement $C \hat{D}(\zeta)$, with $\zeta=g \alpha t$. Interestingly, the controlled-displacement gate becomes both faster and more accurate as we increase $\alpha$, and thus the bias of the ancilla.

We note that to implement the optimized measurement circuit in Fig. 4(b), as well as the optimized stabilizer protocols in Ref. [31], one also requires a rotation gate of the form $\hat{R}_{x}=\exp \left(-i \pi \hat{\sigma}_{x} / 2\right)$. This gate is conveniently very simple to implement for a Kerr-cat qubit. One simply turns off the two-photon pump used to stabilize the Kerr-cat logical subspace, and let the cat evolve freely under the Kerr Hamiltonian $\hat{H}_{K}=-K \hat{a}^{\dagger 2} \hat{a}^{2}$ for a time $\pi / 2 K[49,62]$. One might worry that this gate is not bias preserving: A phase flip prior to, or during, this gate, can be rotated to a bitflip error. However, the circuit in Fig. 4(b) is constructed such that the error that propagates back to the GKP mode is precisely the logical operator we are trying to measure. Similarly, for the protocols in Ref. [31] the error is a stabilizer. This is acceptable, and we therefore expect that these improved measurement and stabilizer protocols also benefit from a biased noise qubit.

Physically, the Kerr-cat qubit can be implemented in a tunable nonlinear element such as capacitively shunted SNAIL, or "SNAILmon" [49]. With an external magnetic flux, the SNAIL exhibits both three-wave and four-wave mixing capabilities. Moreover, the controlleddisplacement Eq. (25), which requires a three-wave mixing interaction between the cat, GKP, and a microwave pump, can also be activated using the same nonlinear element, thus requiring only capacitive coupling between the SNAIL device and the GKP mode [see Fig. 9(b)]. Such an interaction between a Kerr-cat and an unencoded oscillator has been demonstrated [49]. This proof-of-principle demonstration strongly suggests that the Kerr-cat in a SNAILmon can be used for fault-tolerant GKP state preparation. Nonetheless, the viability of this approach depends on the effect of magnetic flux, required to bias the SNAIL, on the lifetime of three-dimensional (3D) cavities and requires more experimental exploration [63]. Alternatively, an approach based on high- $Q$ oscillators in a two-dimensional (2D) architecture must be developed. Finally, we note that this is exactly the same type of interaction required for two-qubit gates between GKP mode, as discussed in Sec. II D3 [c.f. Eq. (23)]. It is thus be possible to repurpose the same piece of hardware for both gates and state preparation [see also Fig. 6(a)]. (a)

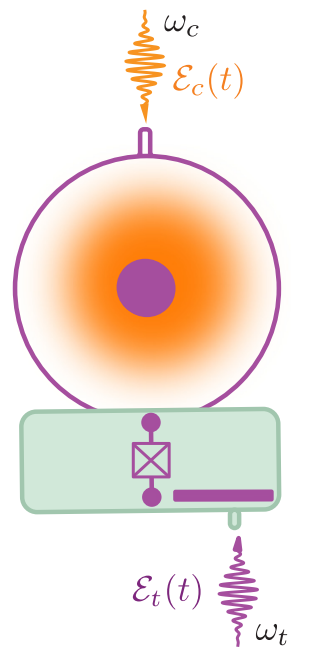

(b)

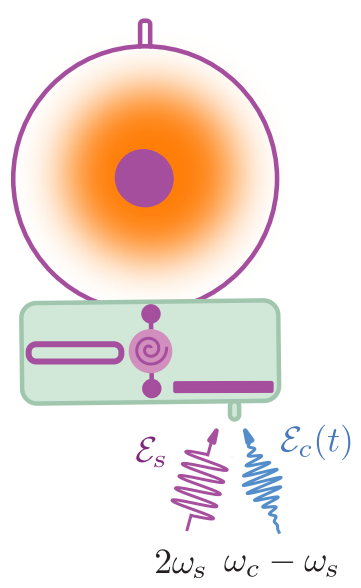

FIG. 9. Implementation of controlled-displacement gate, $C \hat{D}(\zeta)$, between a GKP resonator and a transmon (a) and Kerrcat realized in a SNAIL (b). The frequencies of the resonator, transmon, and SNAIL are $\omega_{c}, \omega_{t}$, and $\omega_{s}$, respectively. The desired effective interaction for $C \hat{D}$ gate is $\propto\left(\hat{a}^{\dagger}+\hat{a}\right) \hat{Z}$, where $\hat{Z}$ is the Pauli operator of the transmon or Kerr-cat qubit. The scheme shown in (a) is from Ref. [3] and uses the cross-Kerr coupling between the transmon and the resonator $=\chi \hat{a}^{\dagger} \hat{a} \hat{Z}$. The underlying principle of the scheme is as follows. In the presence of the microwave pulse $\mathcal{E}_{c}(t)$, the Hamiltonian of the system (in a frame rotating at $\omega_{c}$ ) is $\hat{H}=\chi \hat{a}^{\dagger} \hat{a} \hat{Z}+\mathcal{E}_{c}(t)\left(\hat{a}^{\dagger}+\hat{a}\right)$. In a displaced frame the effective Hamiltonian becomes $\hat{D}(\alpha) \hat{H} \hat{D}^{\dagger}(\alpha)=\chi \hat{a}^{\dagger} \hat{a} \hat{Z}+\chi\left(\hat{a} \alpha^{*}+\hat{a}^{\dagger} \alpha\right) \hat{Z}+\chi|\alpha|^{2} \hat{Z}$, where $\alpha=$ $\int \mathcal{E}_{c}(t) d t$. Clearly, we obtain the important interaction for the $C \hat{D}(\zeta)$ gate given by the second term in this equation. In order to cancel the effect of the other terms, the qubit can be flipped in the middle using the resonant $\pi$ pulse $\mathcal{E}_{t}$. For more details see Ref. [3]. In (b), a Kerr-cat qubit is realized in the SNAIL flux biased at a point where both third- and fourth-order nonlinearities are large. To realize the qubit Hamiltonian, a microwave drive $\mathcal{E}_{s}$ of frequency $2 \omega_{s}$ is applied to the SNAIL. Another microwave drive at frequency $\left|\omega_{c}-\omega_{s}\right|$ is applied to generate Eq. (25). Due to the three-wave mixing, a photon from the latter drive is consumed to convert a photon at the SNAIL to that in the resonator. This effectively creates the desired coupling $\propto\left(\hat{a}^{\dagger}+\hat{a}\right) \hat{Z}$ between the resonator and the Kerr-cat qubit [53].

\section{Error correction with GKP ancillae}

In Sec. IIC we briefly discuss the ability of the GKP code to correct against realistic noise processes in principle by looking at the quantum-error-correction criteria for displacement errors and performing numerical simulations using an optimal recovery map. Performing error correction in practice requires a fault-tolerant and nondestructive way to measure the stabilizers of the GKP code. One way to do this is to perform phase estimation using a two-state ancilla, as we already outline above. However, this approach has the disadvantage that a single bit of information is obtained per ancilla measurement, 
(a)

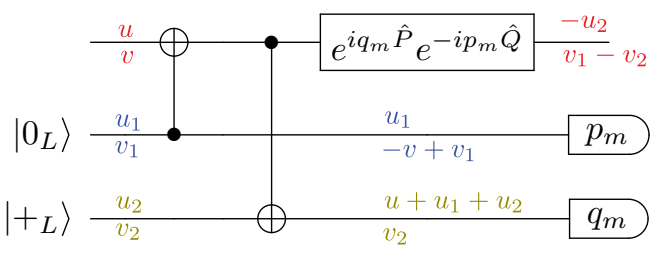

(b)

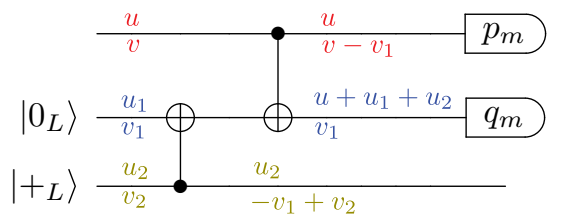

FIG. 10. Illustration of "Steane" (a) and "Knill" (b) errorcorrection circuits. The labels $\{u, v\}$ next to a rail indicates a general displacement error $e^{-i u \hat{P}} e^{i v \hat{Q}}$, and the diagram indicates how the incoming errors propagate through the circuit. The two measurements are of the $\hat{P}$ and $\hat{Q}$ quadratures of the GKP code, respectively, and for the correction shifts one uses the measurement outcomes modulo the GKP lattice spacing $\sqrt{\pi}[1]$.

such that several measurements is required to obtain the continuous-variable GKP syndrome information with high accuracy. An alternative approach, which is the approach that has been studied most extensively in the theoretical literature on GKP codes $[1,22,23,25]$, is to use ancillae that are themselves prepared in GKP states. Here, phase estimation can be performed in a single shot (assuming we can perform high-efficiency homodyne detection), with an accuracy set by the quality of the encoded GKP ancilla states (and the measurement efficiency).

There are two canonical ways to perform "singleshot" GKP error correction using GKP-encoded ancillae. These are essentially bosonic versions of Steane [64] and Knill [65] error correction, respectively. The two schemes are illustrated in Fig. 10. As shown in the figure, the propagation of displacement errors through the two circuits are essentially equivalent, but the Knill circuit does not require any active recovery. Here, the measurements, which contain the syndrome information, are simply used to determine whether a logical Pauli operator has been applied to the GKP state in the process of teleporting from the top to the bottom rail of the circuit [29]. It is not necessary to physically apply any Pauli correction, as it can be tracked in a Pauli frame [65]. We also note that a version of the Knill circuit can be performed using beam-splitter interactions in place of $\bar{C}_{X}$ gates [15], and that it was shown in Refs. $[17,25]$ that this leads to a lower probability of logical error.

Although the "single-shot" GKP error-correction schemes are highly efficient in principle, they also have two clear practical drawbacks: They require preparation of two additional GKP states per round of error correction, and they require very high-efficiency quadrature measurement to be useful. The former itself requires repeated stabilizer measurements using a two-level ancilla as discussed in Sec. III A, unless some other method is developed, and as discussed in Sec. II D, the latter is a highly nontrivial task that has not yet been demonstrated on a GKP state. In the next section, we discuss different approaches to quantum error correction with GKP codes in a large-scale architecture.

\section{THE BIG PICTURE: SCALABILITY AND FAULT TOLERANCE}

So far, we have seen that either due to finite squeezing, environmental noise, or backaction from the ancilla used in state preparation, the logical error rate in the GKP codespace cannot be decreased arbitrarily. In order to correct for residual errors, the GKP code can be concatenated with another binary quantum-error-correcting code. A particularly popular approach is concatenation of GKP code to the topological surface code [66]. Fault-tolerant error correction with GKP-surface codes are being studied in the context of both gate-based [22-25] and measurementbased quantum computing [16,30,67-70]. Here, we limit our discussion to the former as it is more commonly used in the context of the superconducting-circuit and trapped-ion platforms.

In this approach, each data qubit of the surface code is replaced by a single-mode GKP code. Such concatenation provides two layers of protection. In the first layer (referred from henceforth as the inner code and denoted $\mathcal{C}_{\mathrm{GKP}}$ ), the stabilizers $\hat{S}_{X}$ and $\hat{S}_{Z}$ are measured for each GKP mode $M$ times using additional ancillae. These additional ancillae can also be GKP encoded, as in Sec. III C, or discrete qubits such as transmons or Kerr-cats, as discussed in Sec. III B. The ancilla measurement record and details of the underlying noise model are used to estimate and correct, as accurately as possible, the noise on each GKP data mode $[22,23,25]$.

Of course, this procedure will not perfectly remove all errors. The remaining errors are instead corrected by interspersing the stabilizer measurements of $\mathcal{C}_{\mathrm{GKP}}$ with the parity checks of the surface code (referred to henceforth as the outer code, and denoted $\left.\mathcal{C}_{\text {surface }}\right)$. The surface-code ancillae used to measure these parity checks can themselves be GKP encoded or discrete qubits.

This approach of concatenating $\mathcal{C}_{\mathrm{GKP}} \triangleright \mathcal{C}_{\text {surface }}$ may seem contrary to the hardware efficiency of GKP codes argued in the Introduction. After all, at the end we are resorting to a binary surface code, which incurs a substantial hardware overhead (the surface code has a vanishing code rate). Nevertheless, if it becomes possible to suppress the probability of error during logical operations far below the threshold of $\mathcal{C}_{\text {surface, }}$ a modest code distance might suffice to reach a target logical error rate required for useful 


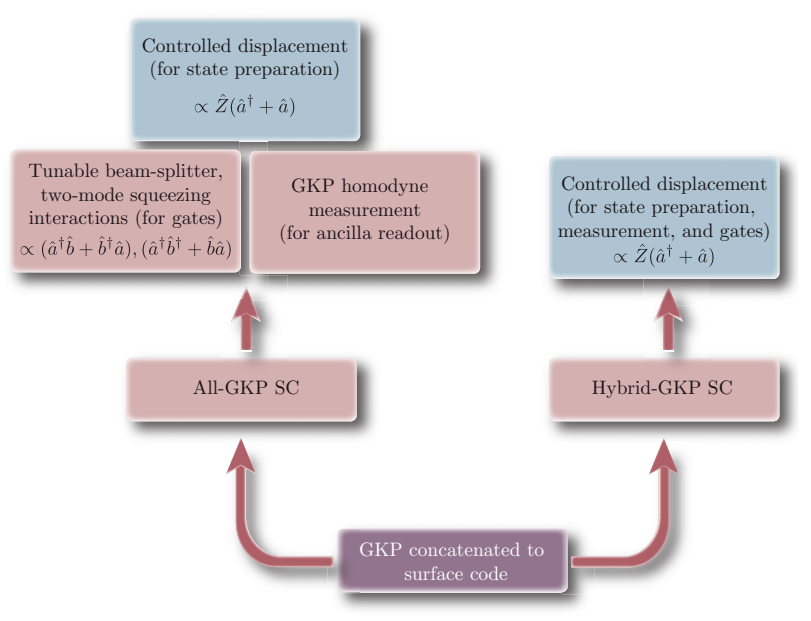

FIG. 11. Building blocks of $\mathcal{C}_{\mathrm{GKP}} \triangleright \mathcal{C}_{\text {surface }}$ with All-GKP surface code and Hybrid-GKP surface code. Of the required operations, only the controlled-displacement gate between a discrete qubit and a GKP-encoded qubit have been demonstrated experimentally $[2,3]$. Not shown in the figure above are single-qubit gates and measurement of the regular ancilla qubits that may be required. We assume these are readily available.

quantum computation [25]. In this case, the resource overhead of quantum hardware and software controls may be significantly lighter than if $\mathcal{C}_{\text {surface }}$ is directly implemented with conventional qubits such as transmons.

With this overview, we now give more details on two possible constructions of the concatenated $\mathcal{C}_{\mathrm{GKP}} \triangleright \mathcal{C}_{\text {surface }}$ code. In one approach, which is also the most widely studied in the theoretical literature, and is referred to as the All-GKP surface code, all the data qubits and ancillae used for error correction are GKP encoded [22,23,25]. The other approach takes a hybrid route where the ancillae are replaced by discrete qubits (e.g., transmons or Kerrcats), and is referred to as the Hybrid-GKP surface code (the two schemes were referred to as Only-Surface-CodeGKP-Ancilla and All-Regular-Qubit-Ancilla, respectively, in Ref. [24]). Figure 11 outlines the building blocks of the two schemes.

\section{A. All-GKP surface code}

A possible layout of $\mathcal{C}_{\mathrm{GKP}} \triangleright \mathcal{C}_{\text {surface }}$ with GKP-encoded data and ancilla modes in the CQED architecture is illustrated in Fig. 12(a). Steane- or Knill-based error-correction circuits may be used for $\mathcal{C}_{\mathrm{GKP}}$ (c.f. Fig. 10), although the former is studied more widely. To be concise, we focus on the square-lattice GKP code, where the probability of $\bar{X}$ errors equals that of $\bar{Z}$ errors, concatenated with the CSS surface code with the "rotated" layout of Ref. [71].

The parity-check operators for $\mathcal{C}_{\text {surface }}$ are of two types: all $\bar{X}$ type to detect $\bar{Z}$ errors and all $\bar{Z}$ type to detect $\bar{X}$ errors. In order to minimize amplification of displacement errors, the surface-code check operators can be modified as shown in Fig. 12(b) [23]. Each $\bar{X}$-type parity check involves two $\bar{X}$ and two $\bar{X}^{\dagger}$, while each $\bar{Z}$-type parity check involves only $\bar{Z}$. (Note in contrast to regular discrete qubits we do not have $\bar{X}=\bar{X}^{\dagger}$ and $\bar{Z}=\bar{Z}^{\dagger}$ outside the GKP codespace). The parity checks of $\mathcal{C}_{\text {surface }}$ are performed simultaneously using the circuit shown in Fig. 12(b). As illustrated, each GKP ancilla (shown in blue in Fig. 12) interacts with four neighboring GKP modes via $\bar{C}_{Z}=e^{i \hat{Q} \otimes \hat{Q}}$ gates for the $Z$ checks and a mix of $\bar{C}_{X}=$ $e^{-i \hat{Q} \otimes \hat{P}}$ and $\bar{C}_{X}^{\dagger}=e^{i \hat{Q} \otimes \hat{P}}$ for the $X$ checks. As discussed earlier, these gates can be implemented via a nonlinear coupler such as a transmon or SNAIL.

Standard techniques of decoding, for example minimumweight perfect matching (MWPM), can be used for error correction in the surface-code layer. Moreover, the accuracy of the decoder can be enhanced by using analog information from the continuous-variable measurement outcomes [22-25,67-70]. For example, one can incorporate the conditional probabilities for Pauli errors given analog measurement outcomes into the edge weights of the MWPM problem, resulting in a dynamic matching graph.

Various independent studies have been performed for estimating the performance of the All-GKP surface code under slightly different assumptions about noise [22-25]. A common feature of most of these studies is that, in order to simplify numerical analysis, noise in approximate GKP states as well as errors introduced during errorcorrection operations are modeled as independent Gaussian displacements with standard deviation $\sigma$, represented by the channel

$$
\mathcal{E}(\hat{\rho})_{\sigma}=\frac{1}{2 \pi \sigma^{2}} \int d^{2} \zeta e^{-|\zeta|^{2} / 2 \sigma^{2}} \hat{D}(\zeta) \hat{\rho} \hat{D}^{\dagger}(\zeta)
$$

Compared to a general noise channel as in Eq. (15), this channel is strictly diagonal in the displacement operator basis. An arbitrary noise channel can be brought closer to diagonal form in the displacement basis using displacement twirling (but not necessarily following a Gaussian distribution) [72]. Equation (26) describes, for example, a noise process with equal loss and heating rates, given by a master equation $\dot{\hat{\rho}}=\kappa\left(\mathcal{D}[\hat{a}] \hat{\rho}+\mathcal{D}\left[\hat{a}^{\dagger}\right] \hat{\rho}\right)[23,35]$. However, in a realistic system special engineering is required to make these noise processes equal. The Gaussian displacement channel does not in general represent physical noise commonly encountered in oscillator systems, such as loss, dephasing, or heating at arbitrary rates.

The standard deviation $\sigma$ in Eq. (26) determines the amount of noise in the system. Similarly to how we introduced a squeezing parameter to quantify the quality of approximate GKP states in Sec. II B, we can introduce a squeezing parameter $\mathcal{S}=-10 \log _{10}\left(2 \sigma^{2}\right)$ (with the identification $\Delta^{2}=2 \sigma^{2}$ ) to quantify the noise in Eq. (26), with large $\mathcal{S}$ meaning low noise. In the numerical studies in Refs. [22,23,25], approximate GKP states with squeezing 

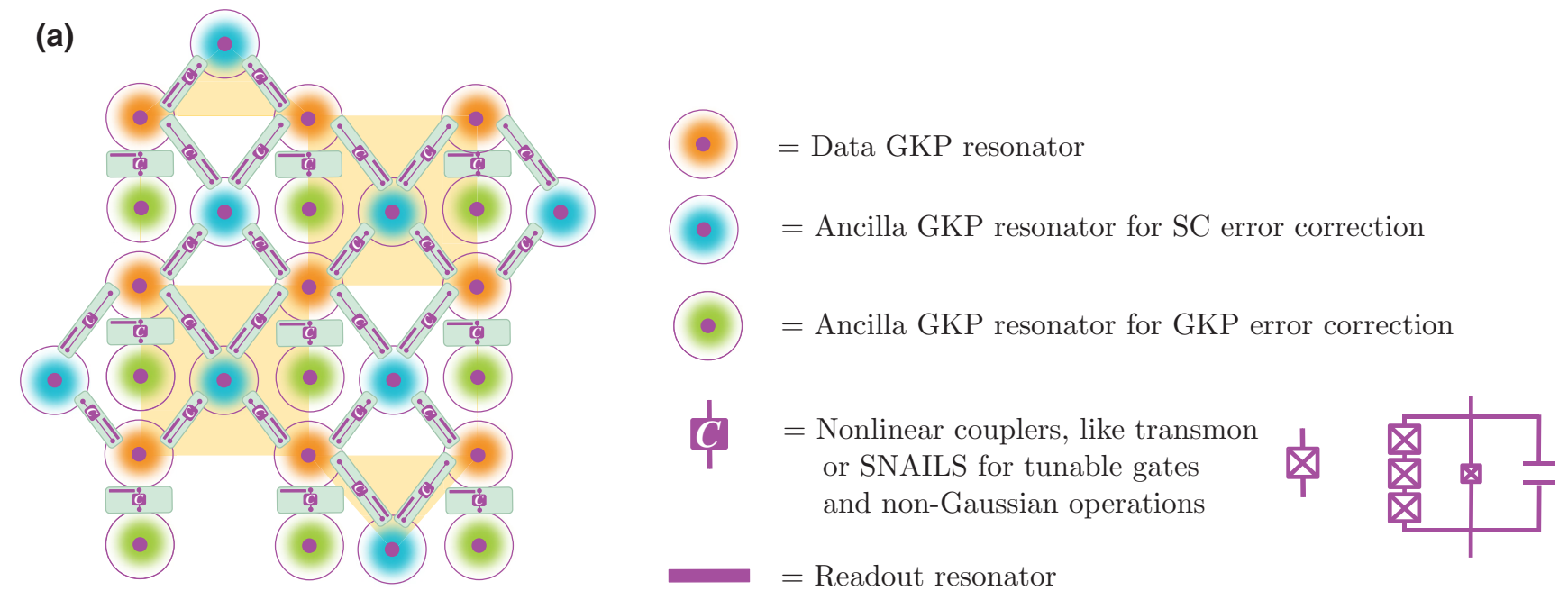

(b)

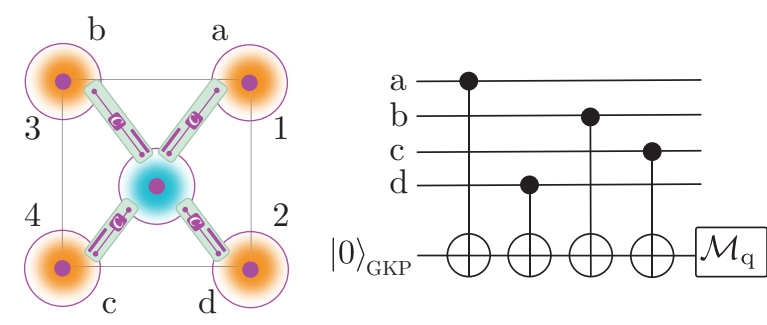

$Z$-stabilizer measurement

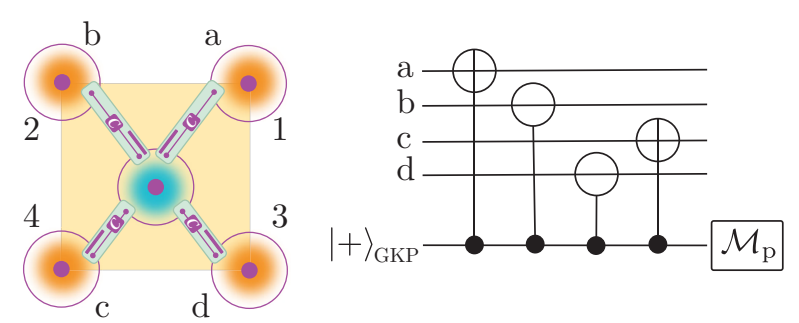

$X$-stabilizer measurement

FIG. 12. (a) Illustration of possible realization of $\mathcal{C}_{\mathrm{GKP}} \triangleright \mathcal{C}_{\text {surface }}$ in cQED. The yellow and white regions indicate $X$ and $Z$ stabilizer checks of the surface code. Scaling up an architecture based on high- $Q 3 \mathrm{D}$ microwave resonators is still a very active area of research. It is also possible to have high- $Q$ resonators on chip as an alternative to the $3 \mathrm{D}$ approach. The purpose of this figure is to indicate the resources that would be required to implement the widely studied All-GKP surface code. Resonators for GKP states used as data qubits in the surface code are indicated in orange. The ancilla GKP states required for realizing Steane-based $\mathcal{C}_{\mathrm{GKP}}$ and $\mathcal{C}_{\text {surface }}$ are shown in green and blue, respectively. State preparation, gates, and measurements are mediated by nonlinear couplers, such as a transmon or a SNAILmon. (b) Stabilizer measurement circuits and gate ordering for surface-code error correction. The controlled- $\ominus$ gate represents the $\bar{C}_{X}^{\dagger}$ gate between the ancilla and data GKP states.

$\Delta$ were modeled by applying the noise channel Eq. (26) with $\sigma=\Delta / \sqrt{2}$ to an ideal GKP state. This was done in order to make the numerics tractable, but the noisy GKP states defined in this manner are still unphysical (there is no "envelope" in phase space). In Ref. [24] it was shown that this model with incoherent displacements underestimates the logical error compared to the coherent superposition in Eq. (10) with $\Delta^{2}=2 \sigma^{2}$.

When the Gaussian displacement channels introducing errors in the GKP codewords and every element (i.e., gates and measurements) of the error-correction circuits are assumed to be equally noisy, and Steane-based error correction is used for $\mathcal{C}_{\mathrm{GKP}}$, then the threshold standard deviation for displacement errors has been found to be $\mathcal{S}=18.6 \mathrm{~dB}$. That is, it becomes possible to realize a logical qubit with arbitrarily small probability of error with $\mathcal{C}_{\mathrm{GKP}} \triangleright \mathcal{C}_{\text {surface }}$ as long as the standard deviation for displacement errors is smaller than approximately 0.09 .
On the other hand, if the Gaussian displacement channel is only applied to the data and ancilla GKP codewords, while all other operations are assumed to be noiseless, this threshold is reduced to $\mathcal{S}=11.2 \mathrm{~dB}$ [23].

Further optimizations are possible, and a recent study showed that the latter threshold with only state-preparation noise can be reduced to $\mathcal{S}=9.9 \mathrm{~dB}$ through better decoding and an optimized error-correction protocol [25]. Beyond improving the threshold, it was shown that a decoding strategy that makes better use of the ana$\log$ syndrome information can have a dramatic effect on the overhead cost to reach a certain logical error rate.

The largest GKP state prepared experimentally so far has a squeezing of approximately $10 \mathrm{~dB}$ and experimental limitations on the performance of two-qubit gates and measurement (c.f. Fig. 3) is largely an open question. Thus, there is opportunity for theoretical and experimental 
innovations in developing scalable quantum-control methods for practical implementation of the GKP-surface code architecture.

One possible path towards easing the threshold requirements is by tailoring the GKP code and the surface code to exploit structure in the noise. In particular, the more recently tailored surface code (TSC) $[73,74]$ and the XZZX surface code [75] exhibit ultrahigh thresholds when the noise channel of the underlying elementary qubits is biased. Recall the rectangular-lattice GKP states introduced in Sec. II A and defined in Eq. (7b). Due to the phase-space structure of the rectangular lattice, the resulting Pauli errors become asymmetric even if the translation errors from the environment are isotropic resulting in a biased noise channel. If $\lambda>1$ in Eq. (7b), then displacements along the momentum quadrature $\hat{P}$ is more likely to be misidentified than displacement along the position quadrature $\hat{Q}$. Consequently, after $M$ rounds of GKP error correction $\bar{X}, \bar{Y}$ errors will be far less likely than $\bar{Z}$ errors. Thus, the error channel after $\mathcal{C}_{\text {gkp }}$ with a rectangular-GKP state will be biased with the bias increasingly exponentially with $\lambda$ [76]. Now, the biased noise can be more accurately corrected if $\mathcal{C}_{\mathrm{GKP}}$ is concatenated to the TSC or the XZZX surface code. A recent work has studied the rectangular-GKP concatenated to the TSC under a simplistic noise model where only the data GKP codewords are subject to a Gaussian displacement channel, while the ancillas and error-correction circuits are perfect [76]. It is known that with such a simplistic noise model, the threshold standard deviation when using the square lattice GKP code is $2.3 \mathrm{~dB}$. If instead a rectangular GKP code is used with $r=\lambda^{2}=3$, then as shown in Ref. [76], the threshold standard deviation increases and corresponds to a squeezing of approximately $1.7 \mathrm{~dB}$. Here, $r=3$, corresponds to a single-mode squeezing of $10 \log _{10}(r) \sim 4.8 \mathrm{~dB}$. For the regular CSS surface code, another study in contrast found only a marginal improvement in logical error when the ancilla (but not data) qubits are rectangular [25]. However, more theoretical work is required to predict if the rectangular-GKP concatenated to the TSC, XZZX, or another code optimized to exploit noise bias, provides a practical advantage when realistic circuit-level noise is considered.

\section{B. Hybrid-GKP surface code}

The All-GKP surface-code scheme may become resource intensive because auxiliary qubits are required to prepare the GKP data and ancilla modes, and additional tunable nonlinear couplers are required to implement two-qubit gates between these GKP modes. Each $\mathcal{C}_{\mathrm{GKP}}$ and $\mathcal{C}_{\text {surface }}$ syndrome measurement moreover requires preparation of fresh GKP ancilla states, which is a slow process [3]. Such extra space-time costs can overwhelm savings in overheads that one may have otherwise expected. A more efficient approach may be to replace some or all of the ancillae with discrete qubits such as a transmon or a biased Kerr-cat qubit (see Sec. III B). As an illustrative example, consider Fig. 12 for counting hardware resources. In this set-up a distance $d$ surface code requires $3 d^{2}-1$ high- $Q$ resonators, and $5 d^{2}-4 d$ nonlinear couplers. Contrast this with $d^{2}$ high- $Q$ cavities and $2 d^{2}-1$ nonlinear couplers that would be required to build a Hybrid-GKP surface code with GKP-encoded data qubits and discrete qubit (Kerr-cat or transmon) ancillae.

Let us now look at the operations required to implement such a Hybrid-GKP surface code. Recall that the controlled-displacement gate $C \hat{D}(\zeta)$ is required to measure the GKP stabilizers. The same gate can also be used to implement the controlled-Pauli gates between the regular qubit ancilla and GKP codewords required for surface-code parity measurements.

Consider, for example, the $\bar{Z}$-type check operators. It can be measured with a discrete ancilla qubit initialized in the $|+\rangle$ state, followed by $C \hat{D}(\zeta)$ gates between the GKP data modes and the ancilla with $\zeta=\beta$, and finally an $X$-basis measurement of the ancilla. This can also be understood as one phase-estimation round of the surfacecode stabilizer, and in analogy with Eq. (18), the probability to get an $X= \pm 1$ outcome is

$$
P( \pm)=\frac{1}{2}\left[1 \pm \frac{1}{2}\left(\langle\bar{Z} \bar{Z} \bar{Z} \bar{Z}\rangle+\left\langle\bar{Z}^{\dagger} \bar{Z}^{\dagger} \bar{Z}^{\dagger} \bar{Z}^{\dagger}\right\rangle\right)\right] .
$$

Clearly, for ideal GKP states [Eq. (6)], $P(+)=1,0$ and $P(-)=0,1$ when $\langle\bar{Z} \bar{Z} \bar{Z} \bar{Z}\rangle= \pm 1$, respectively, and hence this procedure can be used for $\bar{Z}$-parity checks. In the case of approximate GKP states, however, a single round of phase estimation cannot perfectly estimate $\langle\bar{Z} \bar{Z} \bar{Z} \bar{Z}\rangle$, which leads to measurement errors even when the ancilla is noiseless. For small $\Delta$ (see Sec. II B) this measurement error is $p_{\text {err }} \sim\left(1-e^{-\pi \Delta^{2}}\right) / 2 \simeq \pi \Delta^{2} / 2$ [24]. The $\bar{X}$-type parity checks are measured analogously using the $C \hat{D}(\zeta)$ gate with $\zeta=\alpha$. This measurement error can in principle be reduced using the same approach as discussed in Sec. II D2 [see Fig. 4(b)] to give $p_{\text {err }} \sim 0.8 \Delta^{6}$ (in the small $\Delta$ limit) $[31,43]$.

Compared to the All-GKP scheme, where GKP-encoded ancillae are used, the discrete-qubit ancillae may lead to higher-fidelity parity checks when a realistic homodyne measurement efficiency is taken into account. To illustrate, consider a GKP state with approximately $14 \mathrm{~dB}$ of squeezing $(\Delta=0.2)$. In Sec. II D1 we saw that with a measurement efficiency of $\eta=75 \%$ (which is still optimistically high for cQED), the error in the direct homodyne measurement of the ancilla GKP state is approximately $4 \%$. On the other hand, with a discrete qubit ancilla and modified phase-estimation circuit of Ref. [43], we have $p_{\text {err }} \sim 0.0025 \%$. Of course, the ancilla readout itself is not perfect, and errors must be added to $p_{\text {err }}$ to estimate 
the total error in the surface-code parity check. For an ancilla such as a transmon, readout error probability < $2 \%$ is standard, even with $\eta<75 \%[39,77,78]$. Thus, the total measurement infidelity with the discrete qubit ancilla (approximately $0.0025 \%+2 \% \simeq 2 \%$ ) can still be lower than that with a GKP-encoded ancilla.

The hardware simplicity of the Hybrid-GKP surfacecode architecture, which requires only $C \hat{D}(\zeta)$ gates and standard qubit operations, makes this approach very attractive. Nonetheless, a challenge with the hybrid-GKP approach is to prevent fatal propagation of errors from the standard qubit ancilla to the encoded GKP states. Indeed, due to this effect, the current performance of the controlled-displacement gate, the building block of the hybrid scheme, is limited by the relaxation time, $T_{1}$ of the transmon ancilla $[3,62]$. This limitation indicates that it will not be possible to increase the lifetime of a GKP codeword much beyond the $T_{1}$ of the transmon. Fortunately, it is possible to overcome this challenge by replacing the transmon with a biased-noise ancilla such as a Kerr-cat, as we discuss in Sec. III B. This promises a hardwareefficient solution to the problem of ancilla-induced errors and motivates further study to quantify the performance of the hybrid setup.

Finally, it is not necessary that all the ancillae in the GKP-surface code are of the same kind, that is, all GKP encoded or all discrete qubit ancillae. Another possibility is that both the data and syndrome qubits of $\mathcal{C}_{\text {surface }}$ are GKP-encoded, while discrete qubit ancillae are used to perform the $\mathcal{C}_{\mathrm{GKP}}$ stabilizer measurements and phase estimation on the GKP-encoded syndrome qubits. Depending on the properties and performance of operations, we may have an optimized code where some ancillae are discrete qubits while the others are GKP encoded.

\section{Universality}

So far, we have restricted the discussion to error correction in the $\mathcal{C}_{\mathrm{GKP}} \triangleright \mathcal{C}_{\text {surface }}$ code, but do not discuss how universal computation may be performed in this concatenated architecture. An attractive feature of the surface code is that all logical Clifford operations can be implemented via lattice surgery requiring only (single- or two-qubit) logical Pauli measurements $[16,79,80]$. In surface codes with regular qubits, these measurements require nearest-neighbor, physical controlled-Pauli gates between ancilla and data qubits [80]. We have seen how to implement controlledPauli gates between two GKP codewords or between a GKP codeword and a discrete qubit [see Eq. (23), Figs. 6 and 9]. Hence, we can also employ lattice surgery for implementing logical Clifford gates in the $\mathcal{C}_{\mathrm{GKP}} \triangleright \mathcal{C}_{\text {surface }}$ code.

Combined with state preparation of GKP magic states $\left|A_{L}\right\rangle=\bar{T}\left|+_{L}\right\rangle$, logical injection and distillation [81,82] (both of which only require error correction and Clifford

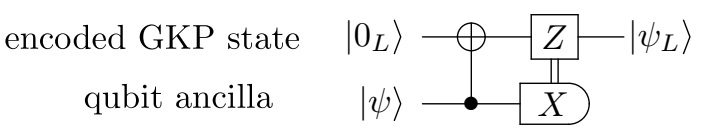

FIG. 13. One-bit teleportation circuit to teleport an arbitrary state from a two-level ancilla (bottom) to an encoded GKP state (top). The $\hat{C}_{X}$ gate is implemented by a controlled displacement $C \hat{D}(\alpha)$. This can be used for preparation of arbitrary states, including magic states, assuming we have universal control over the ancilla.

gates) provides the ability to perform universal quantum computation in the GKP-concatenated surface code. There are several ways to prepare GKP magic states, including using only GKP Pauli states and vacuum as a resource by exploiting the continuous variable nature of the state space [83], but the simplest approach is to use a one-bit teleportation circuit as shown in Fig. 13, which allows us to teleport an arbitrary state from a two-level ancilla to the GKP code.

\section{SUMMARY AND OUTLOOK}

Implementation of the GKP code was once considered, by many, to be beyond impossible. As pointed out by Daniel Gottesman at the Byron Bay Quantum Workshop in 2020 - a workshop dedicated to the 20th anniversary of the GKP code - the authors were aware that the main challenge was going to be the first step of realizing the codewords themselves and the subsequent steps of realizing gates, measurements, etc. would be comparatively simpler. Technological developments since 2001 have made error correction with the GKP code a reality, and this success has inspired more exotic strategies for error correction $[84,85]$. Keeping current and near-future technology in mind, in this Perspective, we explore the prospect of scalable, fault-tolerant quantum error correction with GKP states in a cQED architecture. The most intriguing open question in this direction is whether error correction with GKP states can be made more resource efficient in practice compared to schemes based on conventional qubits. Below we summarize some open theoretical and experimental challenges that must be addressed to answer this question.

One must develop high-fidelity operations, including state-preparation, multiqubit gates, and measurement for GKP states. The fidelity must be better than those of conventional unencoded qubits in the same platform. At the very least, the fidelity should not be limited by decoherence in the auxiliary discrete qubits used for initialization or couplers used for gates. We identify three central challenges in this respect, that can serve as milestones on the path towards a scalable and hardware-efficient quantum computer with GKP-encoded qubits: 
1. State preparation: One must be able to prepare approximate GKP codewords with a sufficiently small $\Delta$, and ensure that the probability of logical errors on the GKP code, e.g., propagating from the ancilla qubit used in the state preparation, is exceedingly low. Since the goal is to outperform the best physical qubit alternatives, specifically transmons and trapped ions, the probability of a logical error in state preparation must be low compared to error rates in these systems. In our view, a biased noise ancilla, such as a Kerr-cat qubit, is promising in this respect, but further analysis is needed to quantify the quality of the GKP states that can be prepared with this approach.

2. Gates: To be able to implement high-fidelity gates as discussed in Sec. II D3 in practice, there are several targets that must be met simultaneously. One must be able to implement pristine two-mode Hamiltonians of the form $\hat{H}_{\theta, \phi} \propto e^{i \theta} \hat{a} \hat{b}^{\dagger}+e^{i \phi} \hat{a} \hat{b}+$ H.c., while keeping any spurious nonlinear terms minimal, and moreover, the two-mode interaction must be switched from near zero to a sufficiently large value such that the gates are fast compared to all decoherence rates. It is crucial that performing these gates does not introduce errors that the GKP code is poor against. In particular, while the GKP is expected to be excellent against loss (and heating), this is not necessarily true for other natural types of noise, such as dephasing (c.f. Fig. 2) and spurious nonlinearities.

3. Measurements: As we have shown in Sec. IID1, a standard homodyne measurement is unlikely to be sufficiently high fidelity to give GKP codes an advantage. Here, further ideas are needed. Either the effective homodyne measurement efficiency has to be increased (probably past $90 \%$ ) using an amplification step prior to release to a standard microwave measurement chain, or one can follow the route of performing phase estimation with a discrete qubit ancilla. It remains to be seen how low the GKP measurement error can be made in practice.

Along with measurement and control, efforts must be devoted to developing technology for scaling up either a $3 \mathrm{D}$ cQED architecture, or an architecture based on high$Q$ resonators on chip. There are also open theoretical questions about how to design such a large-scale architecture. We discuss two different approaches at a high level, the All-GKP surface code and the Hybrid-GKP surface code. Several numerical studies have been performed on the All-GKP surface code, showing promising thresholds and subthreshold behavior [22-25]. However, the noise models used in these studies are rather unrealistic, and more work is needed to model realistic noise accurately. For the Hybrid-GKP surface code, although we think it is quite promising in terms of hardware efficiency, very little quantitative analysis has been done, and its potential is largely unexplored at this stage. Arguably, the most pertinent question here is whether one will ultimately be limited by the discrete qubit ancillae used to stabilize the GKP-encoded qubits and perform syndrome extraction, and consequently whether the Hybrid-GKP approach can have a significant advantage over a more conventional scheme using only discrete qubits everywhere. For example, if biased noise qubits are used as ancillae, one should note that an approach based on using such qubits as both ancillae and data qubits also appears very promising [86-88].

There are further avenues of research we do not touch on in this Perspective, but that nonetheless seem very promising. Alternatively to active GKP error correction, it may be advantageous to explore passive error-correction techniques where the GKP states are stabilized via Hamiltonian engineering $[72,89,90]$. One can also consider alternative concatenation schemes. For example, surface-code variants tailored to the specific noise structure of the GKP codewords may be used. In particular, biased-noise tailored surface code such as the XZZX surface code [75] or tailored surface code $[73,74,76]$, are promising candidates for a rectangular-lattice or squeezed GKP code. More work is required to estimate the performance of such an architecture when realistic circuit-level noise is considered. Other topological codes might offer some advantages over the surface code when it comes to logical gates. For example, the 2D color code has a transversal set of singlequbit Clifford gates. As we show that single-qubit Clifford gates on GKP codes can be done in software, this implies that logical single-qubit Clifford gates on the color code level can be done in software as well. This may lead to some overhead savings for lattice surgery [80,91]. In general, one should follow the design principle of tailoring the overall fault-tolerant scheme to exploit the strengths of the underlying elementary qubits, and further research in this direction is warranted. Finally, concatenation with conventional quantum-error-correcting codes is not the only path towards scalability. It is possible that there is a better scheme where $k$ logical qubits are encoded in $n$ physical modes more directly, i.e., without concatenation with a binary code. Only a small number of works have explored this avenue so far $[1,92,93]$.

With challenges, come opportunities, and with the accelerating pace of technological and theoretical developments the future looks bright for practical quantum computation with GKP codes.

\section{ACKNOWLEDGMENTS}

S.P. thanks numerous colleagues and students, including Steve Girvin, Michel Devoret, Nicholas Frattini, and Alec Eickbusch, discussions and debates with whom have 
shaped her outlook on bosonic codes. In particular, discussions with Nicholas Frattini have helped in producing the conceptual figure for the GKP-surface code. A.L.G. thanks Mackenzie Shaw for deriving the set of generalized control gates for GKP codes, Baptiste Royer for in-depth discussions on preparation of GKP codewords, Ben Baragiola and Joshua Combes for help producing Fig. 1 and input on Sec. II, and Barbara Terhal for several discussions on GKP codes that have informed this Perspective. We thank Baptiste Royer, Alec Eickbusch, and Nicholas Frattini for critical feedback on the Perspective. S.P. is supported by the Army Research Office (ARO) under Grant No. W911NF-18-1-0212. A.L.G. is supported by the Australian Research Council, through the Centre of Excellence for Engineered Quantum Systems (EQUS) Project No. CE170100009 and Discovery Early Career Research Award Project No. DE190100380.

[1] D. Gottesman, A. Kitaev, and J. Preskill, Encoding a qubit in an oscillator, Phys. Rev. A 64, 012310 (2001).

[2] C. Flühmann, T. L. Nguyen, M. Marinelli, V. Negnevitsky, K. Mehta, and J. P. Home, Encoding a qubit in a trapped-ion mechanical oscillator, Nature 566, 513 (2019).

[3] P. Campagne-Ibarcq, A. Eickbusch, S. Touzard, E. ZalysGeller, N. E. Frattini, V. V. Sivak, P. Reinhold, S. Puri, S. Shankar, R. J. Schoelkopf, et al., Quantum error correction of a qubit encoded in grid states of an oscillator, Nature 584, 368 (2020).

[4] N. Ofek, A. Petrenko, R. Heeres, P. Reinhold, Z. Leghtas, B. Vlastakis, Y. Liu, L. Frunzio, S. Girvin, L. Jiang, et al., Extending the lifetime of a quantum bit with error correction in superconducting circuits, Nature 536, 441 (2016).

[5] L. Hu, Y. Ma, W. Cai, X. Mu, Y. Xu, W. Wang, Y. Wu, H. Wang, Y. P. Song, C. L. Zou, S. M. Girvin, L.-M. Duan, and L. Sun, Quantum error correction and universal gate set operation on a binomial bosonic logical qubit, Nat. Phys. 15, 503 (2019).

[6] R. W. Heeres, P. Reinhold, N. Ofek, L. Frunzio, L. Jiang, M. H. Devoret, and R. J. Schoelkopf, Implementing a universal gate set on a logical qubit encoded in an oscillator, Nat. Commun. 8, 1 (2017).

[7] Y. Xu, Y. Ma, W. Cai, X. Mu, W. Dai, W. Wang, L. Hu, X. Li, J. Han, H. Wang, et al., Demonstration of Controlled-Phase Gates Between Two Error-Correctable Photonic Qubits, Phys. Rev. Lett. 124, 120501 (2020).

[8] Y. Ma, Y. Xu, X. Mu, W. Cai, L. Hu, W. Wang, X. Pan, H. Wang, Y. P. Song, C. L. Zou, and L. Sun, Errortransparent operations on a logical qubit protected by quantum error correction, Nat. Phys. 16, 827 (2020).

[9] J. M. Gertler, B. Baker, J. Li, S. Shirol, J. Koch, and C. Wang, Protecting a bosonic qubit with autonomous quantum error correction, Nature 590, 243 (2021).

[10] B. de Neeve, T. L. Nguyen, T. Behrle, and J. Home, Error correction of a logical grid state qubit by dissipative pumping, arXiv:2010.09681 (2020).
[11] I. L. Chuang, D. W. Leung, and Y. Yamamoto, Bosonic quantum codes for amplitude damping, Phys. Rev. A 56, 1114 (1997).

[12] P. T. Cochrane, G. J. Milburn, and W. J. Munro, Macroscopically distinct quantum-superposition states as a bosonic code for amplitude damping, Phys. Rev. A 59, 2631 (1999).

[13] C. Flühmann, V. Negnevitsky, M. Marinelli, and J. P. Home, Sequential Modular Position and Momentum Measurements of a Trapped Ion Mechanical Oscillator, Phys. Rev. X 8, 021001 (2018).

[14] S. Takeda and A. Furusawa, Toward large-scale faulttolerant universal photonic quantum computing, APL Photonics 4, 060902 (2019).

[15] B. W. Walshe, B. Q. Baragiola, R. N. Alexander, and N. C. Menicucci, Continuous-variable gate teleportation and bosonic-code error correction, Phys. Rev. A 102, 062411 (2020).

[16] J. E. Bourassa, R. N. Alexander, M. Vasmer, A. Patil, I. Tzitrin, T. Matsuura, D. Su, B. Q. Baragiola, S. Guha, G. Dauphinais, et al., Blueprint for a scalable photonic fault-tolerant quantum computer, Quantum 5, 392 (2021).

[17] M. V. Larsen, C. Chamberland, K. Noh, J. S. NeergaardNielsen, and U. L. Andersen, A fault-tolerant continuousvariable measurement-based quantum computation architecture, arXiv:2101.03014 (2021).

[18] S. Pirandola, S. Mancini, D. Vitali, and P. Tombesi, Constructing finite-dimensional codes with optical continuous variables, EPL (Europhys. Lett.) 68, 323 (2004).

[19] K. R. Motes, B. Q. Baragiola, A. Gilchrist, and N. C. Menicucci, Encoding qubits into oscillators with atomic ensembles and squeezed light, Phys. Rev. A 95, 053819 (2017).

[20] M. Eaton, R. Nehra, and O. Pfister, Gottesman-KitaevPreskill state preparation by photon catalysis, arXiv:1903. 01925 (2019).

[21] I. Tzitrin, J. E. Bourassa, N. C. Menicucci, and K. K. Sabapathy, Progress towards practical qubit computation using approximate Gottesman-Kitaev-Preskill codes, Phys. Rev. A 101, 032315 (2020).

[22] C. Vuillot, H. Asasi, Y. Wang, L. P. Pryadko, and B. M. Terhal, Quantum error correction with the toric GottesmanKitaev-Preskill code, Phys. Rev. A 99, 032344 (2019).

[23] K. Noh and C. Chamberland, Fault-tolerant bosonic quantum error correction with the surface-GKP code, arXiv:1908.03579 (2019).

[24] B. M. Terhal, J. Conrad, and C. Vuillot, Towards scalable bosonic quantum error correction, Quantum Sci. Technol. 5, 043001 (2020).

[25] K. Noh, C. Chamberland, and F. G. Brandão, Low overhead fault-tolerant quantum error correction with the surfaceGKP code, arXiv:2103.06994 (2021).

[26] W. Cai, Y. Ma, W. Wang, C.-L. Zou, and L. Sun, Bosonic quantum error correction codes in superconducting quantum circuits, Fundam. Res. 1, 50 (2021).

[27] W.-L. Ma, S. Puri, R. J. Schoelkopf, M. H. Devoret, S. Girvin, and L. Jiang, Quantum control of bosonic modes with superconducting circuits, arXiv:2102.09668 (2021).

[28] A. Joshi, K. Noh, and Y. Y. Gao, Quantum information processing with bosonic qubits in circuit QED, Quantum Sci. Technol. 6, 033001 (2021). 
[29] A. L. Grimsmo, J. Combes, and B. Q. Baragiola, Quantum Computing with Rotation-Symmetric Bosonic Codes, Phys. Rev. X 10, 011058 (2020).

[30] N. C. Menicucci, Fault-Tolerant Measurement-Based Quantum Computing with Continuous-Variable Cluster States, Phys. Rev. Lett. 112, 120504 (2014).

[31] B. Royer, S. Singh, and S. M. Girvin, Stabilization of Finite-Energy Gottesman-Kitaev-Preskill States, Phys. Rev. Lett. 125, 260509 (2020).

[32] K. Duivenvoorden, B. M. Terhal, and D. Weigand, Singlemode displacement sensor, Phys. Rev. A 95, 012305 (2017).

[33] M. A. Nielsen and I. L. Chuang, Quantum Computation and Quantum Information (Cambridge University Press, Cambridge, England, 2010).

[34] M. A. Nielsen, A simple formula for the average gate fidelity of a quantum dynamical operation, Phys. Lett. A 303, 249 (2002).

[35] V. V. Albert, K. Noh, K. Duivenvoorden, D. J. Young, R. T. Brierley, P. Reinhold, C. Vuillot, L. Li, C. Shen, S. M. Girvin, B. M. Terhal, and L. Jiang, Performance and structure of single-mode bosonic codes, Phys. Rev. A 97, 032346 (2018).

[36] W. Pfaff, C. J. Axline, L. D. Burkhart, U. Vool, P. Reinhold, L. Frunzio, L. Jiang, M. H. Devoret, and R. J. Schoelkopf, Controlled release of multiphoton quantum states from a microwave cavity memory, Nat. Phys. 13, 882 (2017).

[37] M. H. Shaw and A. L. Grimsmo (to be published).

[38] C. Macklin, K. O’Brien, D. Hover, M. E. Schwartz, V. Bolkhovsky, X. Zhang, W. D. Oliver, and I. Siddiqi, A near-quantum-limited Josephson traveling-wave parametric amplifier, Science 350, 307 (2015).

[39] S. Touzard, A. Kou, N. E. Frattini, V. V. Sivak, S. Puri, A. Grimm, L. Frunzio, S. Shankar, and M. H. Devoret, Gated Conditional Displacement Readout of Superconducting Qubits, Phys. Rev. Lett. 122, 080502 (2019).

[40] A. Eddins, J. M. Kreikebaum, D. M. Toyli, E. M. LevensonFalk, A. Dove, W. P. Livingston, B. A. Levitan, L. C. G. Govia, A. A. Clerk, and I. Siddiqi, High-Efficiency Measurement of an Artificial Atom Embedded in a Parametric Amplifier, Phys. Rev. X 9, 011004 (2019).

[41] B. M. Terhal and D. Weigand, Encoding a qubit into a cavity mode in circuit QED using phase estimation, Phys. Rev. A 93, 012315 (2016).

[42] D. J. Weigand, Ph.D. thesis, Delft University of Technology, Delft, 2020.

[43] J. Hastrup and U. L. Andersen, Improved readout of qubitcoupled Gottesman-Kitaev-Preskill states, arXiv:2008. 10531 (2020).

[44] C. Chamberland, P. Iyer, and D. Poulin, Fault-tolerant quantum computing in the Pauli or Clifford frame with slow error diagnostics, Quantum 2, 43 (2018).

[45] S. Aaronson and D. Gottesman, Improved simulation of stabilizer circuits, Phys. Rev. A 70, 052328 (2004).

[46] D. Gottesman, The Heisenberg representation of quantum computers, arXiv:quant-ph/9807006 (1998).

[47] Y. Y. Gao, B. J. Lester, K. S. Chou, L. Frunzio, M. H. Devoret, L. Jiang, S. M. Girvin, and R. J. Schoelkopf, Entanglement of bosonic modes through an engineered exchange interaction, Nature 566, 509 (2019).
[48] C. S. Wang, J. C. Curtis, B. J. Lester, Y. Zhang, Y. Y. Gao, J. Freeze, V. S. Batista, P. H. Vaccaro, I. L. Chuang, L. Frunzio, L. Jiang, S. M. Girvin, and R. J. Schoelkopf, Efficient Multiphoton Sampling of Molecular Vibronic Spectra on a Superconducting Bosonic Processor, Phys. Rev. X 10, 021060 (2020).

[49] A. Grimm, N. E. Frattini, S. Puri, S. O. Mundhada, S. Touzard, M. Mirrahimi, S. M. Girvin, S. Shankar, and M. H. Devoret, Stabilization and operation of a Kerr-cat qubit, Nature 584, 205 (2020).

[50] A. Roy and M. Devoret, Introduction to parametric amplification of quantum signals with Josephson circuits, C. R. Phys. 17, 740 (2016).

[51] N. Frattini, U. Vool, S. Shankar, A. Narla, K. Sliwa, and M. Devoret, 3-Wave mixing Josephson dipole element, Appl. Phys. Lett. 110, 222603 (2017).

[52] J. Hastrup, K. Park, J. B. Brask, R. Filip, and U. L. Andersen, Measurement-free preparation of grid states, npj Quantum Inf. 7, 1 (2021).

[53] S. Puri, A. Grimm, P. Campagne-Ibarcq, A. Eickbusch, K. Noh, G. Roberts, L. Jiang, M. Mirrahimi, M. H. Devoret, and S. M. Girvin, Stabilized cat in a Driven Nonlinear Cavity: A Fault-Tolerant Error Syndrome Detector, Phys. Rev. X 9, 041009 (2019).

[54] Y. Shi, C. Chamberland, and A. W. Cross, Fault-tolerant preparation of approximate GKP states, arXiv:1905.00903 (2019).

[55] S. Rosenblum, P. Reinhold, M. Mirrahimi, L. Jiang, L. Frunzio, and R. J. Schoelkopf, Fault-tolerant detection of a quantum error, Science 361, 266 (2018).

[56] N. Earnest, S. Chakram, Y. Lu, N. Irons, R. K. Naik, N. Leung, L. Ocola, D. A. Czaplewski, B. Baker, J. Lawrence, J. Koch, and D. I. Schuster, Realization of a $\Lambda$ System with Metastable States of a Capacitively Shunted Fluxonium, Phys. Rev. Lett. 120, 150504 (2018).

[57] A. Gyenis, P. S. Mundada, A. Di Paolo, T. M. Hazard, X. You, D. I. Schuster, J. Koch, A. Blais, and A. A. Houck, Experimental realization of a protected superconducting circuit derived from the $0-\pi$ qubit, PRX Quantum 2, 010339 (2021).

[58] R. Lescanne, M. Villiers, T. Peronnin, A. Sarlette, M. Delbecq, B. Huard, T. Kontos, M. Mirrahimi, and Z. Leghtas, Exponential suppression of bit-flips in a qubit encoded in an oscillator, Nat. Phys. 16, 509 (2020).

[59] C. Siegele, M. Mirrahimi, and P. Campagne-Ibarcq, Faulttolerant error syndrome detection in the GKP code, Bull. Am. Phys. Soc. (2021).

[60] S. Puri, S. Boutin, and A. Blais, Engineering the quantum states of light in a kerr-nonlinear resonator by two-photon driving, npj Quantum Inf. 3, 18 (2017).

[61] G. J. Woeginger, Open problems around exact algorithms, Discrete Appl. Math. 156, 397 (2008).

[62] S. Puri, L. St-Jean, J. A. Gross, A. Grimm, N. E. Frattini, P. S. Iyer, A. Krishna, S. Touzard, L. Jiang, A. Blais, S. T. Flammia, and S. M. Girvin, Bias-preserving gates with stabilized cat qubits, Sci. Adv. 6, eaay5901 (2020).

[63] B. Chapman, S. de Graaf, Y. Zhang, S. Mundhada, L. Frunzio, S. Girvin, and R. Schoelkopf, Mediating high-fidelity interactions between superconducting microwave cavities, part II, Bull. Am. Phys. Soc. (2021). 
[64] A. M. Steane, Active Stabilization, Quantum Computation, and Quantum State Synthesis, Phys. Rev. Lett. 78, 2252 (1997).

[65] E. Knill, Quantum computing with realistically noisy devices, Nature 434, 39 (2005).

[66] E. Dennis, A. Kitaev, A. Landahl, and J. Preskill, Topological quantum memory, J. Math. Phys. (N.Y.) 43, 4452 (2002).

[67] K. Fukui, A. Tomita, and A. Okamoto, Analog Quantum Error Correction with Encoding a Qubit into an Oscillator, Phys. Rev. Lett. 119, 180507 (2017).

[68] K. Fukui, A. Tomita, A. Okamoto, and K. Fujii, HighThreshold Fault-Tolerant Quantum Computation with Analog Quantum Error Correction, Phys. Rev. X 8, 021054 (2018).

[69] K. Fukui, A. Tomita, and A. Okamoto, Tracking quantum error correction, Phys. Rev. A 98, 022326 (2018).

[70] K. Fukui, High-threshold fault-tolerant quantum computation with the GKP qubit and realistically noisy devices, arXiv:1906.09767 (2019).

[71] H. Bombin and M. A. Martin-Delgado, Optimal resources for topological two-dimensional stabilizer codes: Comparative study, Phys. Rev. A 76, 012305 (2007).

[72] J. Conrad, Twirling and hamiltonian engineering via dynamical decoupling for Gottesman-Kitaev-Preskill quantum computing, Phys. Rev. A 103, 022404 (2021).

[73] D. K. Tuckett, S. D. Bartlett, and S. T. Flammia, Ultrahigh Error Threshold for Surface Codes with Biased Noise, Phys. Rev. Lett. 120, 050505 (2018).

[74] D. K. Tuckett, S. D. Bartlett, S. T. Flammia, and B. J. Brown, Fault-Tolerant Thresholds for the Surface Code in Excess of 5\% Under Biased Noise, Phys. Rev. Lett. 124, 130501 (2020).

[75] J. P. B. Ataides, D. K. Tuckett, S. D. Bartlett, S. T. Flammia, and B. J. Brown, The XZZX surface code, Nat. Commun. 12, 1 (2021).

[76] L. Hänggli, M. Heinze, and R. König, Enhanced noise resilience of the surface-Gottesman-Kitaev-Preskill code via designed bias, Phys. Rev. A 102, 052408 (2020).

[77] M. Hatridge, S. Shankar, M. Mirrahimi, F. Schackert, K. Geerlings, T. Brecht, K. Sliwa, B. Abdo, L. Frunzio, S. M. Girvin, et al., Quantum back-action of an individual variable-strength measurement, Science 339, 178 (2013).

[78] T. Walter, P. Kurpiers, S. Gasparinetti, P. Magnard, A. Potočnik, Y. Salathé, M. Pechal, M. Mondal, M. Oppliger, C. Eichler, et al., Rapid High-Fidelity Single-
Shot Dispersive Readout of Superconducting Qubits, Phys. Rev. Appl. 7, 054020 (2017).

[79] C. Horsman, A. G. Fowler, S. Devitt, and R. Van Meter, Surface code quantum computing by lattice surgery, New J. Phys. 14, 123011 (2012).

[80] D. Litinski and F. von Oppen, Lattice surgery with a twist: Simplifying clifford gates of surface codes, Quantum 2, 62 (2018).

[81] S. Bravyi and A. Kitaev, Universal quantum computation with ideal Clifford gates and noisy ancillas, Phys. Rev. A 71, 022316 (2005).

[82] D. Litinski, A game of surface codes: Large-scale quantum computing with lattice surgery, Quantum 3, 128 (2019).

[83] B. Q. Baragiola, G. Pantaleoni, R. N. Alexander, A. Karanjai, and N. C. Menicucci, All-Gaussian universality and fault tolerance with the Gottesman-Kitaev-Preskill code, arXiv:1903.00012 (2019).

[84] V. V. Albert, J. P. Covey, and J. Preskill, Robust Encoding of a Qubit in a Molecule, Phys. Rev. X 10, 031050 (2020).

[85] J. A. Gross, Encoding a qubit in a spin, arXiv:2005.10910 (2020).

[86] J. Guillaud and M. Mirrahimi, Repetition Cat Qubits for Fault-Tolerant Quantum Computation, Phys. Rev. X 9, 041053 (2019), publisher: American Physical Society.

[87] C. Chamberland, K. Noh, P. Arrangoiz-Arriola, E. T. Campbell, C. T. Hann, J. Iverson, H. Putterman, T. C. Bohdanowicz, S. T. Flammia, A. Keller, G. Refael, J. Preskill, L. Jiang, A. H. Safavi-Naeini, O. Painter, and F. G. S. L. Brandão, Building a fault-tolerant quantum computer using concatenated cat codes, arXiv:2012.04108 (2020).

[88] A. S. Darmawan, B. J. Brown, A. L. Grimsmo, D. K. Tuckett, and S. Puri, Practical quantum error correction with the XZZX code and Kerr-cat qubits, arXiv:2104.09539 (2021).

[89] D. T. Le, A. Grimsmo, C. Müller, and T. Stace, Doubly nonlinear superconducting qubit, Phys. Rev. A 100, 062321 (2019).

[90] M. Rymarz, S. Bosco, A. Ciani, and D. P. DiVincenzo, Hardware-Encoding Grid States in a Nonreciprocal Superconducting Circuit, Phys. Rev. X 11, 011032 (2021).

[91] A. J. Landahl and C. Ryan-Anderson, Quantum computing by color-code lattice surgery, arXiv:1407.5103 (2014).

[92] J. Harrington and J. Preskill, Achievable rates for the gaussian quantum channel, Phys. Rev. A 64, 062301 (2001).

[93] K. Noh, V. V. Albert, and L. Jiang, Quantum capacity bounds of gaussian thermal loss channels and achievable rates with Gottesman-Kitaev-Preskill codes, IEEE Trans. Inf. Theory 65, 2563 (2019). 